\title{
Tetrakis[3,5-bis(pentafluorosulfanyl)phenyl]borate - A Weakly Coordinating Anion Probed in Polymerization Catalysis
}

\author{
Supporting Information
}

Daniel Langford§, Inigo Göttker-Schnetmann ${ }^{\S}$, Florian P. Wimmer ${ }^{\S}$, Larissa A. Casper $^{\dagger}$, Philip Kenyon ${ }^{\S}$, Rainer F. Winter ${ }^{\dagger}$ and Stefan Mecking ${ }^{\S}$

$\S$ Chair of Chemical Material Science, Department of Chemistry, University of Konstanz, Universitätsstrasse 10, 78457 Konstanz, Germany

$\uparrow$ Chair of Organometallic $\pi$-Systems, Department of Chemistry, University of Konstanz, Universitätsstraße 10 , 78457 Konstanz, Germany 


\section{Table of Contents}

$1 \quad$ Experimental Section___ S3

1.1 Materials and General Considerations __ S3

1.2 Sodium Tetrakis[3,5-bis(pentafluorosulfanyl)phenyl]borate ___ S4

1.3 Tetrabutylammonium Tetrakis(3,5-bis(pentafluoro-sulfanyl)phenyl)borate __ S5

1.4 Nickel(allyl)(mesitylene) Tetrakis(3,5-bis(pentafluoro-sulfanyl)phenyl)borate __ S6

1.5 Nickel(allyl)(mesitylene) Tetrakis(3,5-bis(trifluoromethyl)-phenyl)borate___ S7

2 Further Characterization Data __ S8

2.1 NMR Spectra of Sodium Tetrakis[3,5-bis(pentafluoro-sulfanyl)phenyl]borate___ S8

2.2 HR-ESI-MS Spectrum of Sodium Tetrakis(3,5-bis(pentafluorosulfanyl)phenyl)borate

2.3 Thermogravimetric Analysis of Sodium Tetrakis(3,5-bis(pentafluorosulfanyl)phenyl)borate

2.4 NMR Spectra of Nickel(allyl)(mesitylene) Tetrakis(3,5-bis(pentafluorosulfanyl)phenyl)borate

2.5 ${ }^{19}$ F NMR Spectra of Experiments with Highly Reactive Transmetalation Reagents

$3 \quad$ Single Crystal Structures

3.1 $\mathrm{Na}(\text { thf })_{5}-\mu-\mathrm{O}-(\mathrm{HO})-\mathrm{B}\left(\mathrm{Ar}^{\mathrm{SF} 5}\right)_{3} \_\mathrm{S} 14$

3.2 Nickel(allyl)(mesitylene) Tetrakis(3,5-bis(pentafluoro-sulfanyl)phenyl)borate _ S16

4 Polymers

4.1 General Procedure for Butadiene Polymerization

4.2 NMR Spectra of Polymers

4.3 GPC Traces of Polymers

4.4 DSC Data of Polymers

5 DFT Calculations

5.1 Computational Details

5.2 Structures

5.3 Graphical Representation of Frontier Orbitals

6 Cyclovoltammetric Measurements S30

7 References 


\section{Experimental Section}

\subsection{Materials and General Considerations}

All experiments were carried out under an inert nitrogen atmosphere using standard Schlenk or glovebox techniques, unless noted otherwise. Glassware was dried at $90{ }^{\circ} \mathrm{C}$ overnight or under vacuum with a heat gun prior use. Diethyl ether and methylene chloride were dried and deoxygenated by passing through columns of $3 \AA$ molecular sieve and BASF R3-11 catalyst. Heptane was distilled from $\mathrm{CaH}_{2}$.

Allylnickel chloride dimer (MCAT) and 1-bromo-3,5-bis(pentafluorosulfanyl)benzene (UBE Industries - Japan) were commercially available and used as received. A 2 M solution of ${ }^{i} \mathrm{PrMgCl}$ in $\mathrm{Et}_{2} \mathrm{O}$, a $1 \mathrm{M}$ solution of $\mathrm{BCl}_{3}$ in heptane and $\mathrm{Na}_{2} \mathrm{CO}_{3}$ were purchased from Sigma-Aldrich and used as received. Sodium tetrakis(3,5-bis(trifluoromethyl)phenyl)borate was prepared according to a reported procedure. ${ }^{1}$ 1,3-Butadiene was purchased from Air Liquide and passed through a drying column prior use.

NMR spectra were recorded on a Bruker Avance III 400 at $300 \mathrm{~K}$ in deuterated solvents purchased from Eurisotop. The spectra were processed using MestReNova. ${ }^{1} \mathrm{H}$ chemical shifts were referenced to the solvents residual ${ }^{1} \mathrm{H}$ signals. ${ }^{13} \mathrm{C}$ chemical shifts were referenced to the solvent signals.

Gel permeation chromatography (GPC) was carried out on a Polymer Laboratories PL-GPC 50 instrument with two PLgel $5 \mu \mathrm{m}$ Mixed-C columns in THF at $40{ }^{\circ} \mathrm{C}$ using refractive index detection vs. poly(styrene) standards, or on a PSS SECcurity ${ }^{2}$ GPC System with two PSS SDV linear M $3 \mu \mathrm{m}$ columns in THF at $35^{\circ} \mathrm{C}$ using refractive index detection vs. poly(styrene) standards. Differential scanning calorimetry measurements were performed on a Netzsch DSC $204 \mathrm{~F} 1$ for determination of the glass transition temperature (cooling to $-150{ }^{\circ} \mathrm{C}$ with $30 \mathrm{~K} / \mathrm{min}$ and heating to $20^{\circ} \mathrm{C}$ with $30 \mathrm{~K} / \mathrm{min}$ ). ESI-MS measurements were performed on a Bruker micrOTOF II with direct injection of the analyte dissolved in acetonitrile.

Thermogravimetric analyses were performed on a Netzsch STA 449 F3 Jupiter with a heating rate of $10{ }^{\circ} \mathrm{C} / \mathrm{min}$ up to $1000{ }^{\circ} \mathrm{C}$ under a nitrogen atmosphere.

Single crystal X-Ray diffraction was performed on a STOE IPDS 2T diffractometer. A suitable crystal was selected under an optical microscope and mounted with silicon grease. The crystal 
was cooled to $100 \mathrm{~K}$ during data acquisition. The structure was solved with the ShelXS 2016/6 software by using direct methods und refined with the ShelXL 2016/6 refinement package using least squares minimization. Graphical representations were created by the ORTEP-3 V2.02 software package.

Cyclic voltammograms were recorded in a custom-built vacuum-tight single compartment cell. Spiral shaped Pt and Ag wires as counter and reference electrodes sealed into glass capillaries are introduced via Quickfit screws at opposite sides of the cell. A Pt electrode is fitted into the cell through the top central port via Teflon screw cap. The Pt working electrode was polished with $1 \mu \mathrm{m}$ and $0.25 \mu \mathrm{m}$ diamond paste (Buehler-Wirtz) before each set of measurements. The cell can be fitted to a conventional Schlenk line via a side port equipped with a Teflon screw valve allowing for measurements under inert gas atmosphere. Data was recorded with a computer-controlled BAS potentiostat. Referencing was performed by adding an equimolar amount of $\left(\mathrm{CpMe}_{5}\right)_{2} \mathrm{Fe}$ as internal standard after acquiring all data of interest. Representative sets of scans were repeated with the added standard. Finally, referencing was performed against the ferrocene/ferrocenium couple.

\subsection{Sodium Tetrakis[3,5-bis(pentafluorosulfanyl)phenyl]borate}

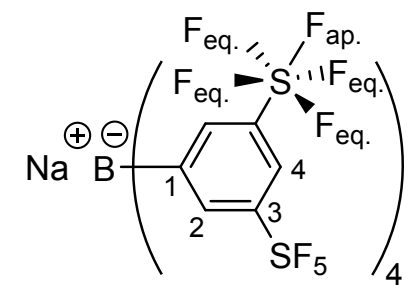

A $100 \mathrm{~mL}$ Schlenk flask was charged with $4.00 \mathrm{~g}$ (9.78 mmol, 6.0 equiv.) of 1-bromo-3,5bis(pentafluorosulfanyl)benzene and $40 \mathrm{~mL}$ of anhydrous $\mathrm{Et}_{2} \mathrm{O}$. The solution was cooled to $-78{ }^{\circ} \mathrm{C}$ and $4.9 \mathrm{~mL}$ (9.80 mmol, 6.0 equiv.) of a $2 \mathrm{M}$ solution of ${ }^{i} \mathrm{PrMgCl}$ in $\mathrm{Et}_{2} \mathrm{O}$ were added dropwise. The mixture was stirred at $-78^{\circ} \mathrm{C}$ for 5 minutes, subsequently for 25 minutes at $0{ }^{\circ} \mathrm{C}$ and then left to warm to room temperature over the course of 60 minutes. The mixture was then cooled to $0{ }^{\circ} \mathrm{C}$ for 10 minutes and $1.63 \mathrm{~mL}$ (1.63 mmol, 1.0 equiv.) of a $1 \mathrm{M}$ solution of $\mathrm{BCl}_{3}$ in heptane was added. The $\mathrm{BCl}_{3}$ solution was injected with a syringe directly into the Grignard solution. The mixture was stirred for 15 minutes at $0{ }^{\circ} \mathrm{C}$, and stirring was continued at room temperature for further 19 hours. A continuous formation of a colorless precipitate was observed during that time. The reaction suspension was added to a solution of $8.00 \mathrm{~g}$ of $\mathrm{Na}_{2} \mathrm{CO}_{3}$ in $500 \mathrm{~mL}$ of deionized $\mathrm{H}_{2} \mathrm{O}$ and stirred vigorously for one hour. The aqueous phase was extracted with $\mathrm{Et}_{2} \mathrm{O}(4 \times 150 \mathrm{~mL})$. The combined organic phases were washed with $250 \mathrm{~mL}$ of 
a concentrated solution of $\mathrm{NaCl}$ in $\mathrm{H}_{2} \mathrm{O}$. The organic phase was dried over $\mathrm{Na}_{2} \mathrm{SO}_{4}$ and filtered over Celite before the solvent was removed under reduced pressure. The solid residue was dissolved in $45 \mathrm{~mL}$ of acetone and a total of $1060 \mathrm{~mL}$ of pentane were added in $20 \mathrm{~mL}$ steps, during which a colorless solid precipitated. The liquid phase was removed via cannula filtration. The solid residue was washed with pentane $(3 \times 50 \mathrm{~mL})$ and dried under vacuum for 20 minutes. The colorless solid was dispersed in $250 \mathrm{~mL}$ of benzene and dried via azeotropic distillation for one hour. The residual benzene was removed with a syringe and the solid was dried in vacuo at $100{ }^{\circ} \mathrm{C}$ over night. Sodium tetrakis(3,5-bis(pentafluorosulfanyl)phenyl)borate was obtained as a colorless solid in $61 \%$ yield $(1.33 \mathrm{~g}, 0.99 \mathrm{mmol})$.

${ }^{1} \mathbf{H}$ NMR $\left(400 \mathrm{MHz}\right.$, acetone- $\left.d_{6}, 300 \mathrm{~K}\right) \delta=8.08\left(\mathrm{~m}, 8 \mathrm{H}, \mathrm{H}^{2}\right), 7.95\left(\mathrm{~m}, 4 \mathrm{H}, \mathrm{H}^{4}\right) \mathrm{ppm}$.

${ }^{13} \mathbf{C}\left\{{ }^{1} \mathbf{H}\right\}$ NMR $\left(100 \mathrm{MHz}\right.$, acetone- $\left.d_{6}, 300 \mathrm{~K}\right) \delta=162.67\left(\mathrm{q},{ }^{1} J_{\mathrm{CB}}=49.9 \mathrm{~Hz}, \mathrm{C}^{1}\right), 153.99(\mathrm{~m}$, $\left.\mathrm{C}^{3}\right), 135.19\left(\mathrm{~s}, \mathrm{C}^{2}\right), 120.73\left(\mathrm{~s}, \mathrm{C}^{4}\right) \mathrm{ppm}$.

${ }^{11} \mathbf{B}\left\{{ }^{1} \mathbf{H}\right\}$ NMR $\left(128 \mathrm{MHz}\right.$, acetone- $\left.d_{6}, 300 \mathrm{~K}\right) \delta=-5.76$ (s) ppm.

${ }^{19} \mathbf{F}\left\{{ }^{1} \mathbf{H}\right\}$ NMR $\left(376 \mathrm{MHz}\right.$, acetone- $\left.d_{6}, 300 \mathrm{~K}\right) \delta=83.24$ (quint, $\left.{ }^{2} J_{\mathrm{FF}}=148.8 \mathrm{~Hz}, \mathrm{~F}_{\mathrm{ap}}\right), 62.43(\mathrm{~d}$, ${ }^{2} J_{\mathrm{FF}}=148.8 \mathrm{~Hz}, \mathrm{~F}_{\mathrm{eq}}$.) ppm.

Calculated Elemental Analysis: C, 21.34\%; H, 0.90\%; S, 18.99\%.

Found: C, 21.26\%; H, 1.44\%; S, approx. 18\%.

Note: The high fluorine content is problematic for elemental analysis.

HR ESI-MS (negative) (m/z): 1326.8281 [M] $^{-}$(calc. $1326.8161[\mathrm{M}]^{-}$), deviation: 9.04 ppm.

\subsection{Tetrabutylammonium Tetrakis(3,5-bis(pentafluoro- sulfanyl)phenyl)borate}

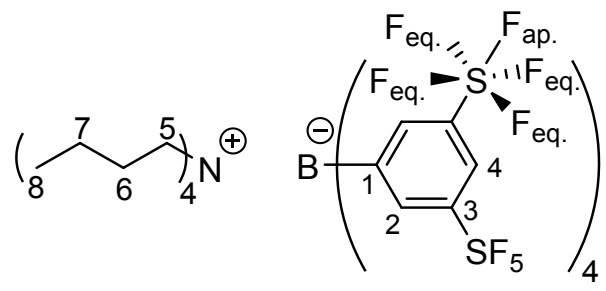

$100 \mathrm{mg}(74 \mu \mathrm{mol})$ sodium tetrakis[3,5-bis(pentafluoro-sulfanyl)phenyl]borate and $24 \mathrm{mg}$ (74 $\mu \mathrm{mol})$ of tetrabutylammoniumbromide were dissolved in a mixture of $3 \mathrm{~mL}$ of methanol and $1.5 \mathrm{~mL}$ of acetone and stirred at room temperature for $1 \mathrm{~h}$. Addition of $2 \mathrm{~mL}$ of water 
precipitated a white solid which was separated by centrifugation and dried over $\mathrm{P}_{4} \mathrm{O}_{10}$ to yield the desired product in quantitative yield as a white powder.

${ }^{1} \mathbf{H}$ NMR (400 MHz, $\mathrm{CD}_{2} \mathrm{Cl}_{2}, 300 \mathrm{~K}$ ) $\delta=7.89$ (vquint, 8H, H²), 7.84 (vt, 4H, $\mathrm{H}^{4}$ ), $3.09-3.02$ $\left(\mathrm{m}, 8 \mathrm{H}, \mathrm{H}^{5}\right), 1.66-1.54\left(\mathrm{~m}, 8 \mathrm{H}, \mathrm{H}^{6}\right), 1.41\left(\mathrm{vsext}, 8 \mathrm{H}, \mathrm{H}^{7}\right), 1.01\left(\mathrm{t},{ }^{3} J_{\mathrm{HH}}=7.1 \mathrm{~Hz}, 12 \mathrm{H}, \mathrm{H}^{8}\right)$ ppm.

${ }^{11} \mathbf{B}\left\{{ }^{1} \mathbf{H}\right\}$ NMR $\left(128 \mathrm{MHz}, \mathrm{CD}_{2} \mathrm{Cl}_{2}, 300 \mathrm{~K}\right) \delta=-5.89 \mathrm{ppm}$.

${ }^{19} \mathbf{F}\left\{{ }^{1} \mathbf{H}\right\}$ NMR $\left(376 \mathrm{MHz}, \mathrm{CD}_{2} \mathrm{Cl}_{2}, 300 \mathrm{~K}\right) \delta=83.73$ (quint, ${ }^{2} J_{\mathrm{FF}}=151.3 \mathrm{~Hz}, \mathrm{~F}_{\mathrm{ap}}$ ), 62.89 (d, ${ }^{2} J_{\mathrm{FF}}=152.4 \mathrm{~Hz}, \mathrm{~F}_{\mathrm{eq}}$.) ppm.

Calculated Elemental Analysis: C, 30.60\%; H, 3.08\%; N, 0.89\%.

Found: C, 30.58\%; H, 3.48\%; N, 1.20 .

Note: The high fluorine content is problematic for elemental analysis.

\subsection{Nickel(allyl)(mesitylene) Tetrakis(3,5-bis(pentafluoro- sulfanyl)phenyl)borate}

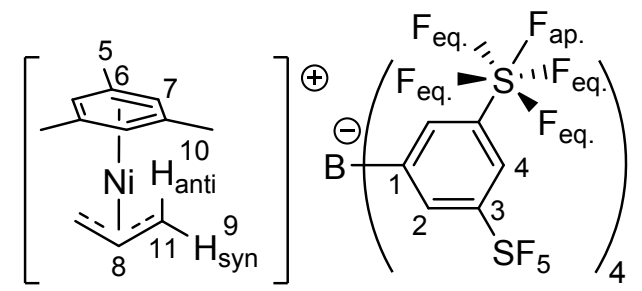

A $25 \mathrm{~mL}$ Schlenk flask was charged with $60.4 \mathrm{mg} \quad(0.223 \mathrm{mmol}, 1.0$ equiv $)$ of allylnickel chloride dimer and $603.3 \mathrm{mg}(0.447 \mathrm{mmol}, 2.0$ equiv) of anhydrous sodium tetrakis(3,5-bis(pentafluorosulfanyl)phenyl)borate. The mixture was cooled to $-78{ }^{\circ} \mathrm{C}$ before $10 \mathrm{~mL}$ of $\mathrm{Et}_{2} \mathrm{O}$ and $0.19 \mathrm{~mL}$ of mesitylene (1.38 mmol, 6.2 equiv.) were added. The resulting dispersion was stirred for 10 minutes at $-78^{\circ} \mathrm{C}$ and for further 60 minutes at $0{ }^{\circ} \mathrm{C}$ before the solvent was removed under reduced pressure at RT. The red residue was redissolved in $20 \mathrm{~mL}$ of DCM, first filtrated over Celite and subsequently using 2 syringe filters $(0.2 \mu \mathrm{m})$. The DCM was removed under reduced pressure at RT. The red residue was washed with pentane $(3 \times 10 \mathrm{~mL})$ and dried in vacuo at RT over night. Nickel(allyl)(mesitylene) tetrakis(3,5-bis(pentafluorosulfanyl)phenyl)borate was obtained as a red solid in $60 \%$ yield (417 mg, $0.269 \mathrm{mmol})$. The product was stored at $-30{ }^{\circ} \mathrm{C}$ under inert nitrogen atmosphere.

${ }^{1} \mathbf{H}$ NMR $\left(400 \mathrm{MHz}, \mathrm{CD}_{2} \mathrm{Cl}_{2}, 300 \mathrm{~K}\right) \delta=7.89\left(\mathrm{~m}, 8 \mathrm{H}, \mathrm{H}^{2}\right), 7.82\left(\mathrm{~m}, 4 \mathrm{H}, \mathrm{H}^{4}\right), 6.70\left(\mathrm{~s}, 3 \mathrm{H}, \mathrm{H}^{7}\right)$, $5.89\left(\right.$ vsept, $\left.{ }^{3} J_{\mathrm{HH}}=12.5 \mathrm{~Hz},{ }^{3} J_{\mathrm{HH}}=6.6 \mathrm{~Hz}, 1 \mathrm{H}, \mathrm{H}^{8}\right), 3.73\left(\mathrm{~d},{ }^{3} J_{\mathrm{HH}}=6.6 \mathrm{~Hz}, 2 \mathrm{H}, \mathrm{H}^{9}{ }_{\text {syn }}\right), 2.48(\mathrm{~d}$, $\left.{ }^{3} J_{\mathrm{HH}}=2.48,2 \mathrm{H}, \mathrm{H}^{10}{ }_{\text {anti }}\right), 2.38\left(\mathrm{~s}, 9 \mathrm{H}, \mathrm{H}^{5}\right) \mathrm{ppm}$. 
${ }^{13} \mathbf{C}\left\{{ }^{1} \mathbf{H}\right\}$ NMR $\left(100 \mathrm{MHz}, \mathrm{CD}_{2} \mathrm{Cl}_{2}, 300 \mathrm{~K}\right) \delta=161.95\left(\mathrm{q},{ }^{1} J_{\mathrm{CB}}=49.9 \mathrm{~Hz}, \mathrm{C}^{1}\right), 153.61\left(\mathrm{~m}, \mathrm{C}^{3}\right)$, $134.61\left(\mathrm{~s}, \mathrm{C}^{2}\right), 125.07\left(\mathrm{~s}, \mathrm{C}^{6}\right), 120.34\left(\mathrm{~s}, \mathrm{C}^{4}\right), 109.70\left(\mathrm{~s}, \mathrm{C}^{7}\right), 107.76\left(\mathrm{~s}, \mathrm{C}^{8}\right), 59.91\left(\mathrm{~s}, \mathrm{C}^{11}\right)$, $20.81\left(\mathrm{~s}, \mathrm{C}^{5}\right) \mathrm{ppm}$.

${ }^{11} \mathbf{B}\left\{{ }^{1} \mathbf{H}\right\}$ NMR $\left(128 \mathrm{MHz}, \mathrm{CD}_{2} \mathrm{Cl}_{2}, 300 \mathrm{~K}\right) \delta=-5.86$ (s) ppm.

${ }^{19} \mathbf{F}\left\{{ }^{1} \mathrm{H}\right\}$ NMR $\left(376 \mathrm{MHz}, \mathrm{CD}_{2} \mathrm{Cl}_{2}, 300 \mathrm{~K}\right) \delta=83.76$ (quint, $\left.{ }^{2} J_{\mathrm{FF}}=149.9 \mathrm{~Hz}, \mathrm{~F}_{\text {ap. }}\right), 62.72(\mathrm{~d}$, ${ }^{2} J_{\mathrm{FF}}=149.9 \mathrm{~Hz}, \mathrm{~F}_{\mathrm{eq}}$.) ppm.

Calculated Elemental Analysis: C, 27.94\%; H, 1.89\%; S, 16.57\%.

Found: C, 28.80\%; H, 2.76\%; S, 16.96\%.

Note: The high fluorine content is problematic for elemental analysis.

\subsection{Nickel(allyl)(mesitylene) Tetrakis(3,5-bis(trifluoromethyl)- phenyl)borate}

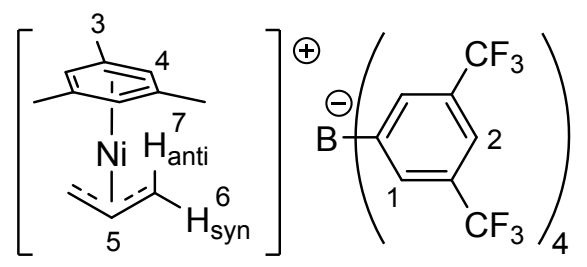

This known compound was prepared according to a reported procedure. ${ }^{2}$ A $25 \mathrm{~mL}$ Schlenk flask was charged with $101.0 \mathrm{mg} \quad(0.373 \mathrm{mmol}, \quad 1.0$ equiv. $)$ of allylnickel chloride dimer and $662.1 \mathrm{mg}(0.747 \mathrm{mmol}, 2.0$ equiv.) of sodium tetrakis(3,5-bis(trifluoromethyl)phenyl)borate. The mixture was cooled to $-78^{\circ} \mathrm{C}$ and $6 \mathrm{~mL}$ of $\mathrm{Et}_{2} \mathrm{O}$ and $0.14 \mathrm{~mL}$ of mesitylene (1.01 mmol, 2.7 equiv.) were added. The mixture was stirred at $-78^{\circ} \mathrm{C}$ for 10 minutes and for further 60 minutes at $0{ }^{\circ} \mathrm{C}$, and the $\mathrm{Et}_{2} \mathrm{O}$ was removed under reduced pressure at RT. The residue was redissolved in $4 \mathrm{~mL}$ of DCM and filtrated over Celite. The DCM was removed under reduced pressure and the residue was washed with pentane (4 $\times 5 \mathrm{~mL})$. After drying in vacuo over night at RT, nickel(allyl)(mesitylene) tetrakis(3,5-bis (trifluoromethyl)phenyl)borate was obtained as a red solid in $43 \%$ yield (347 mg, $0.320 \mathrm{mmol}$ ).

${ }^{1} \mathbf{H}$ NMR $\left(400 \mathrm{MHz}, \mathrm{CD}_{2} \mathrm{Cl}_{2}, 300 \mathrm{~K}\right) \delta=7.72\left(\mathrm{~s}, 8 \mathrm{H}, \mathrm{H}^{1}\right), 7.57\left(\mathrm{~s}, 4 \mathrm{H}, \mathrm{H}^{2}\right), 6.67\left(\mathrm{~s}, 3 \mathrm{H}, \mathrm{H}^{4}\right)$, $5.86\left(\mathrm{vsept},{ }^{3} J_{\mathrm{HH}}=12.4 \mathrm{~Hz},{ }^{3} J_{\mathrm{HH}}=6.6 \mathrm{~Hz}, 1 \mathrm{H}, \mathrm{H}^{5}\right), 3.70\left(\mathrm{~d},{ }^{3} J_{\mathrm{HH}}=6.6 \mathrm{~Hz}, 2 \mathrm{H}, \mathrm{H}^{6}{ }_{\text {syn }}\right), 2.46(\mathrm{~d}$, ${ }^{3} J_{\mathrm{HH}}=12.4 \mathrm{~Hz}, 2 \mathrm{H}, \mathrm{H}^{7}$ anti) $) 2.36\left(\mathrm{~s}, 9 \mathrm{H}, \mathrm{H}^{3}\right) \mathrm{ppm}$. 


\section{Further Characterization Data}

\subsection{NMR Spectra of Sodium Tetrakis[3,5-bis(pentafluoro- sulfanyl)phenyl]borate}

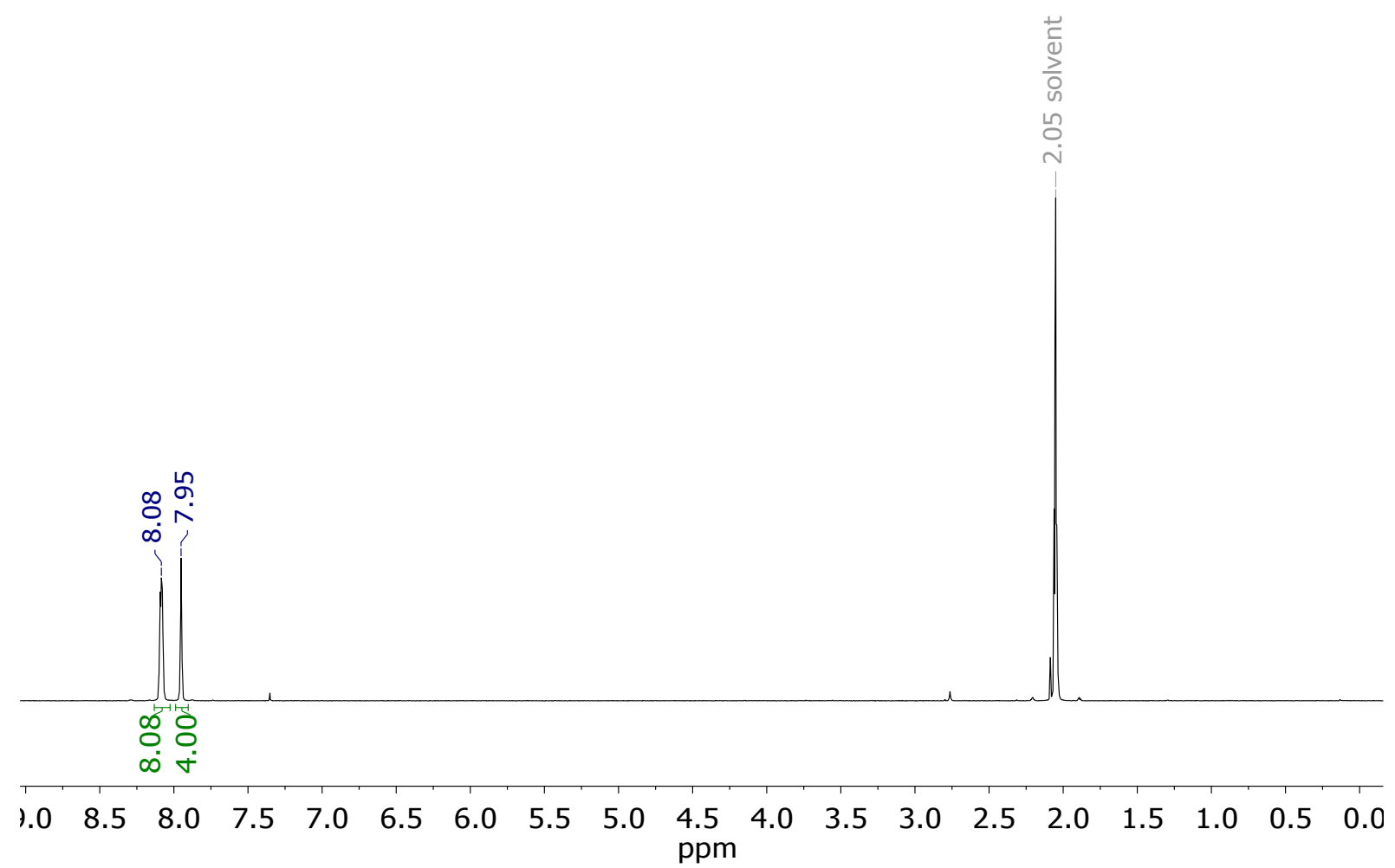

Figure S1. ${ }^{1} \mathrm{H}$ NMR spectrum of sodium tetrakis[3,5-bis(pentafluorosulfanyl)phenyl]borate, recorded in acetone- $d_{6}$ at $300 \mathrm{~K}$.

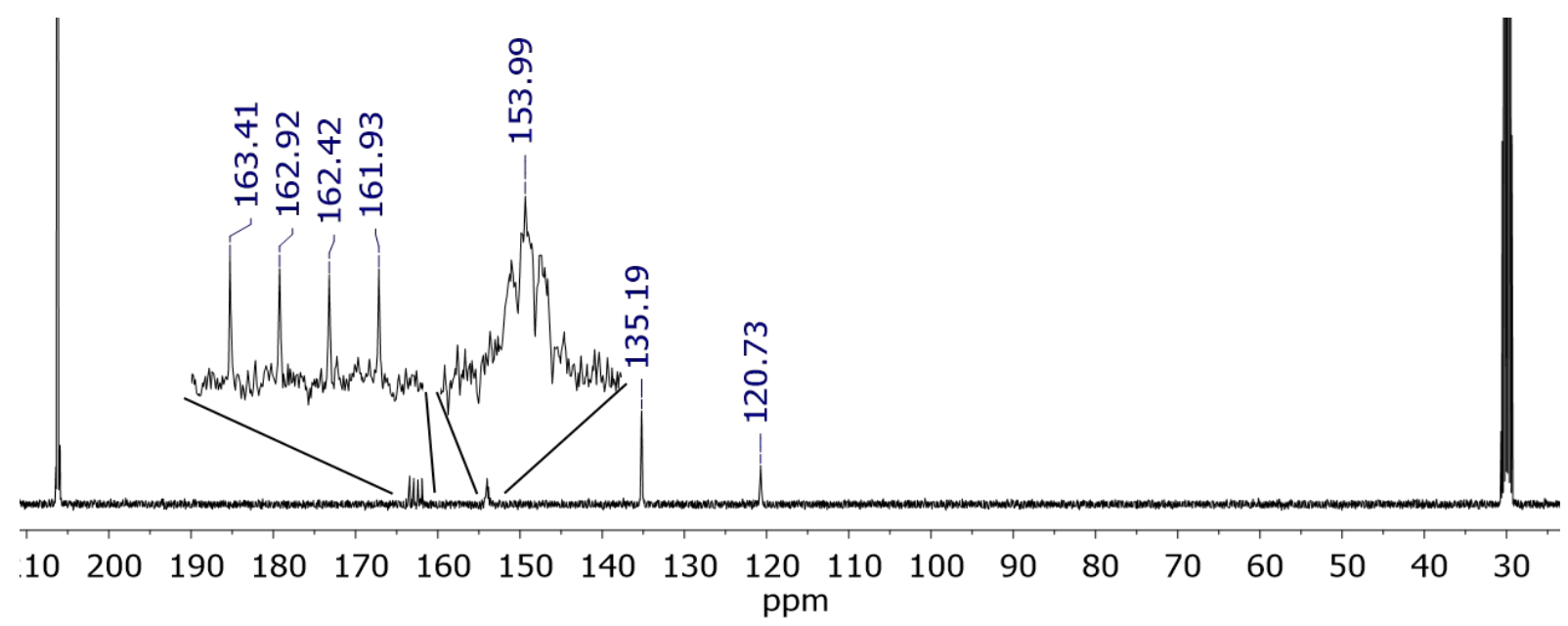

Figure S2. ${ }^{13} \mathrm{C}\left\{{ }^{1} \mathrm{H}\right\}$ NMR spectrum of sodium tetrakis[3,5-bis(pentafluorosulfanyl)phenyl]borate, recorded in acetone- $d_{6}$ at $300 \mathrm{~K}$. 


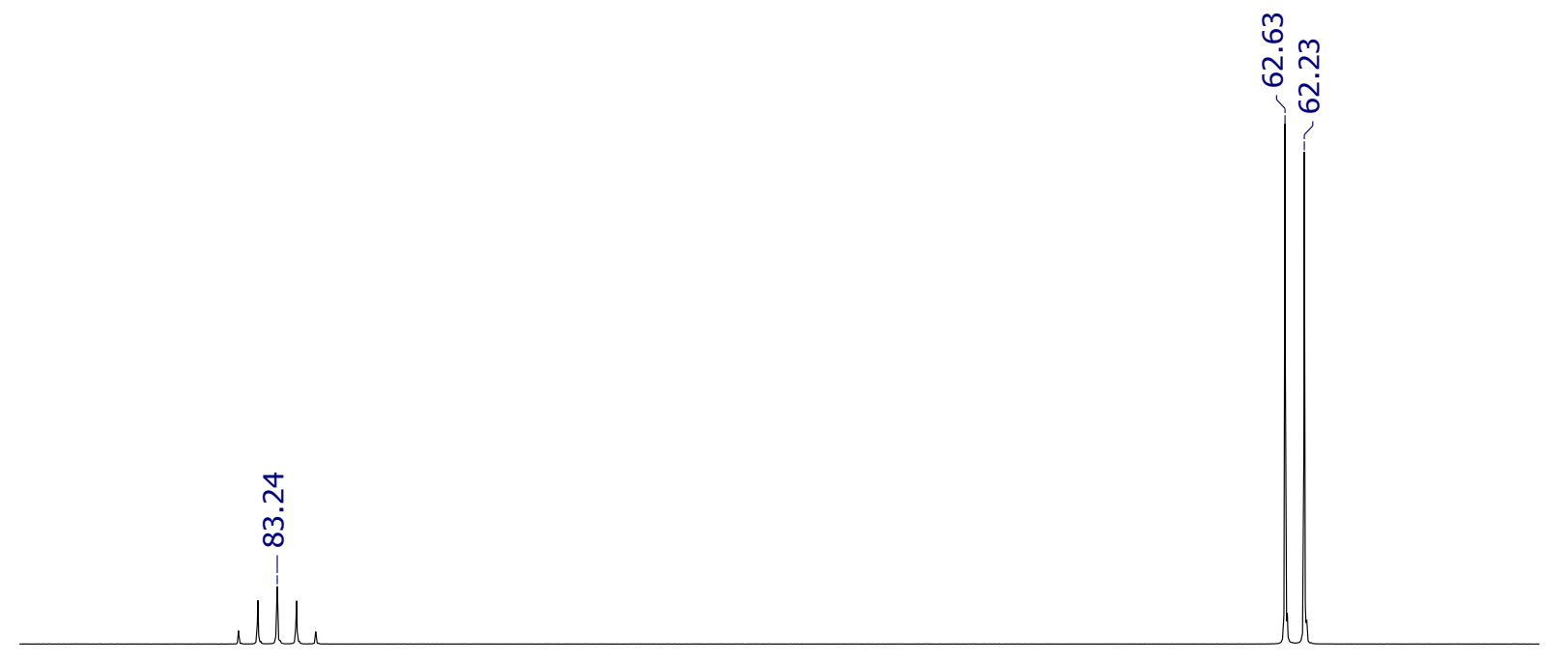

$\begin{array}{lllllllllllllllllllllllllllllll}88 & 87 & 86 & 85 & 84 & 83 & 82 & 81 & 80 & 79 & 78 & 77 & 76 & 75 & 74 & 73 & 72 & 71 & 70 & 69 & 68 & 67 & 66 & 65 & 64 & 63 & 62 & 61 & 60 & 59 & 58\end{array}$ ppm

Figure S3. ${ }^{19} \mathrm{~F}\left\{{ }^{1} \mathrm{H}\right\}$ NMR spectrum of sodium tetrakis[3,5-bis(pentafluorosulfanyl)phenyl]borate, recorded in acetone- $d_{6}$ at $300 \mathrm{~K}$.

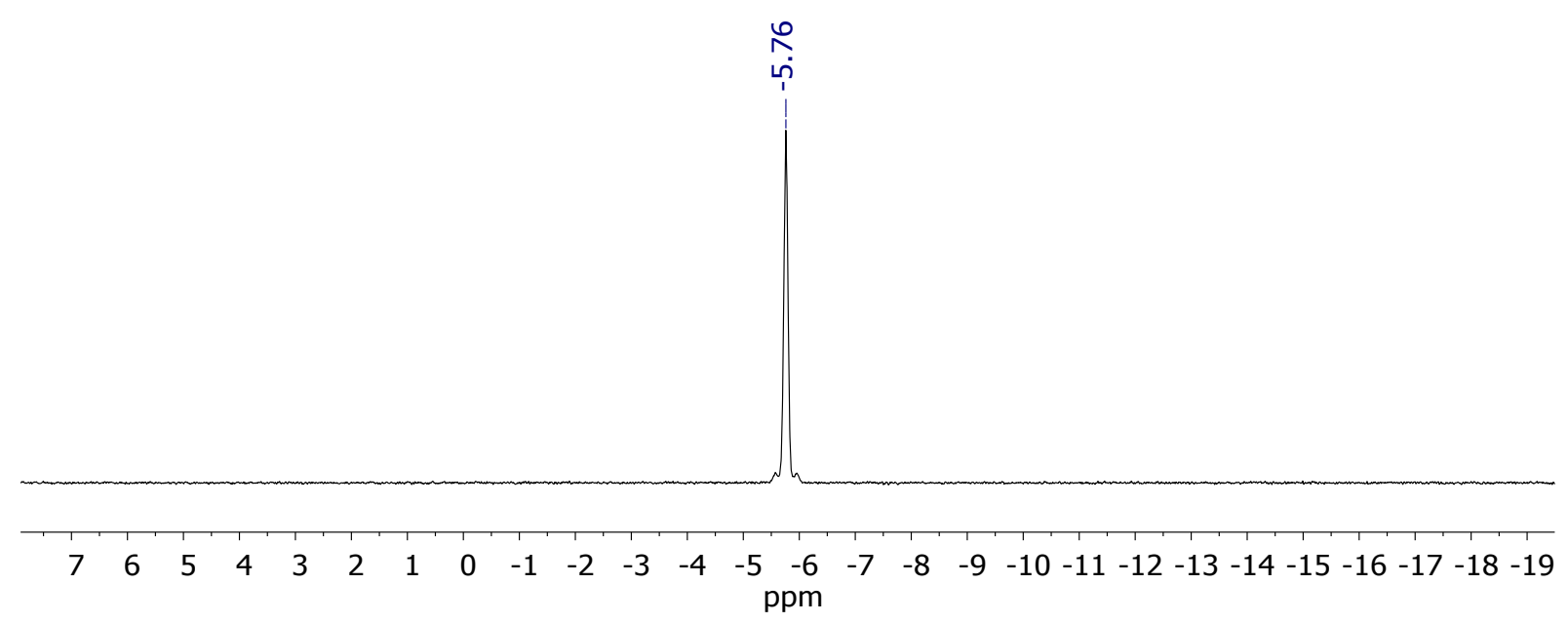

Figure S4. ${ }^{11} \mathrm{~B}\left\{{ }^{1} \mathrm{H}\right\}$ NMR spectrum of sodium tetrakis[3,5-bis(pentafluorosulfanyl)phenyl]borate, recorded in acetone- $d_{6}$ at $300 \mathrm{~K}$. 


\subsection{HR-ESI-MS Spectrum of Sodium Tetrakis(3,5-bis-}

(pentafluorosulfanyl)phenyl)borate

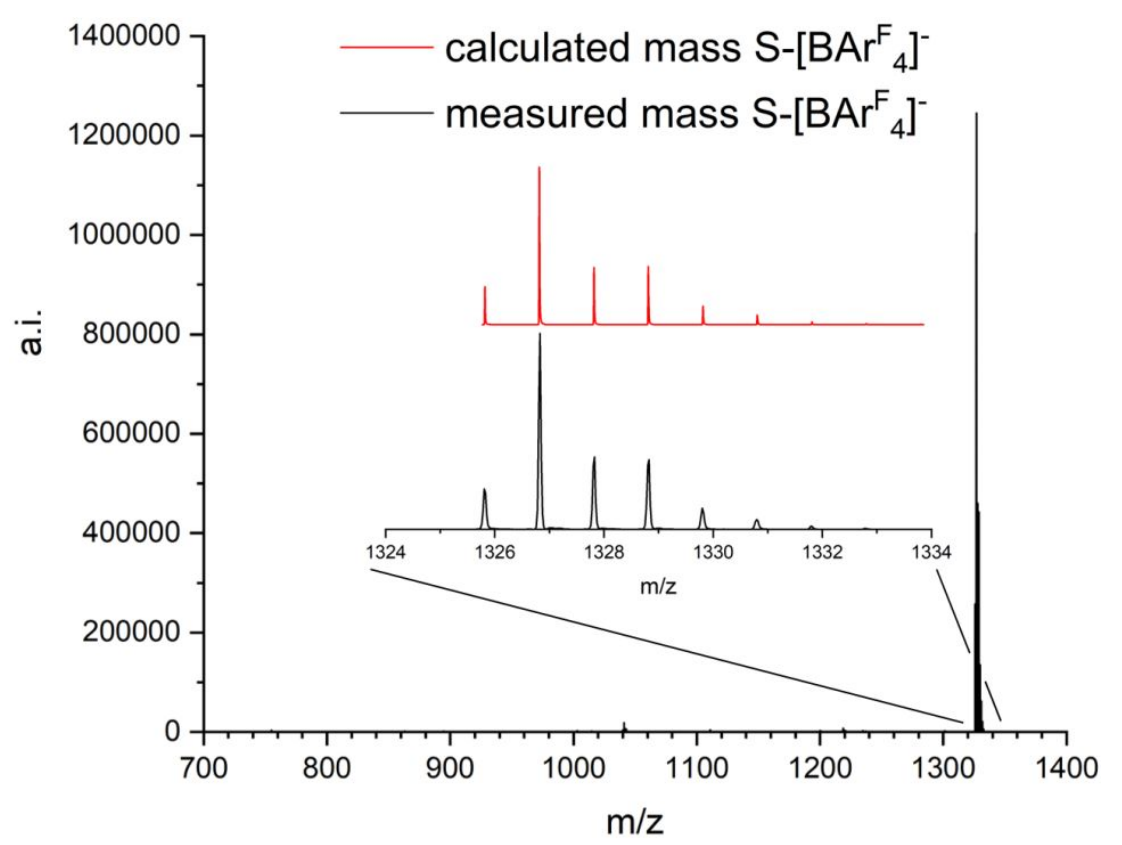

Figure S5. HR ESI-MS spectrum of sodium tetrakis[3,5-bis(pentafluorosulfanyl)phenyl]borate.

\subsection{Thermogravimetric Analysis of Sodium Tetrakis(3,5-bis-} (pentafluorosulfanyl)phenyl)borate

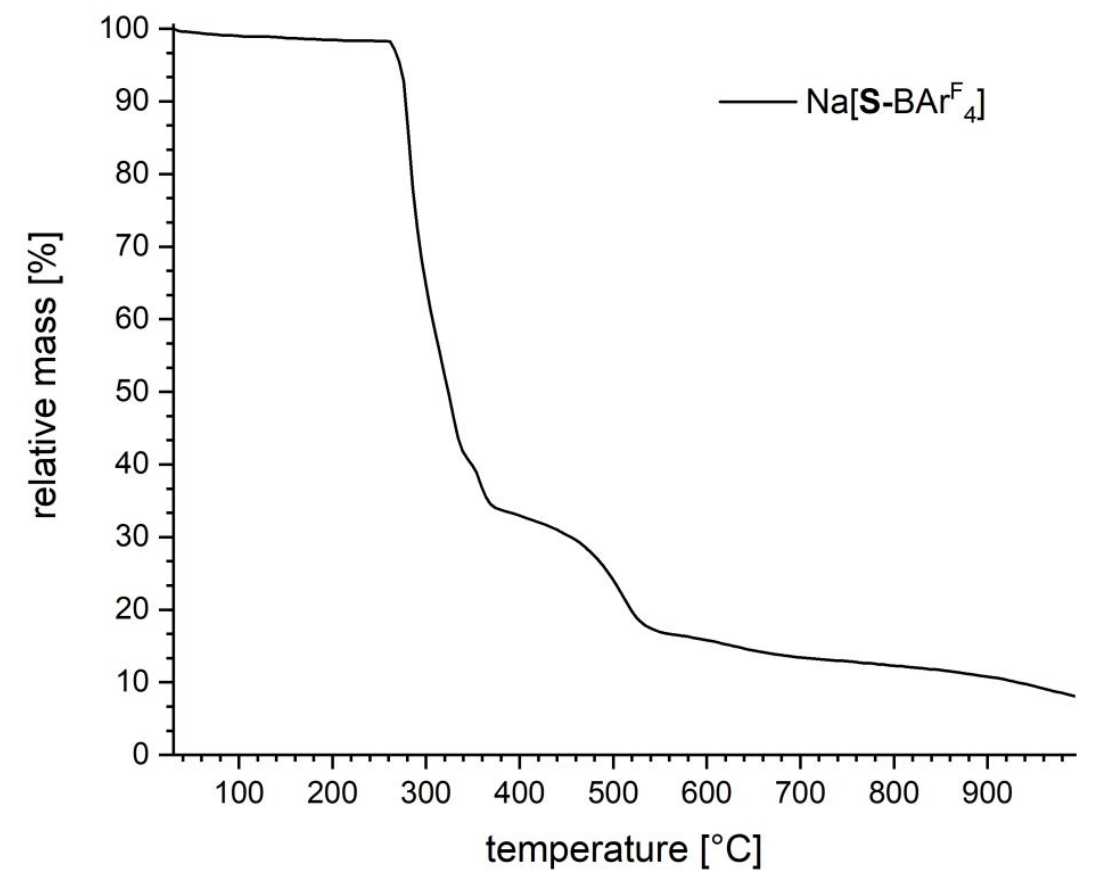

Figure S6. Thermogravimetric analysis of $\mathrm{Na}\left[\mathrm{S}-\mathrm{BAr}{ }_{4}^{\mathrm{F}}\right]$. 


\subsection{NMR Spectra of Nickel(allyl)(mesitylene) Tetrakis(3,5-bis- (pentafluorosulfanyl)phenyl)borate}

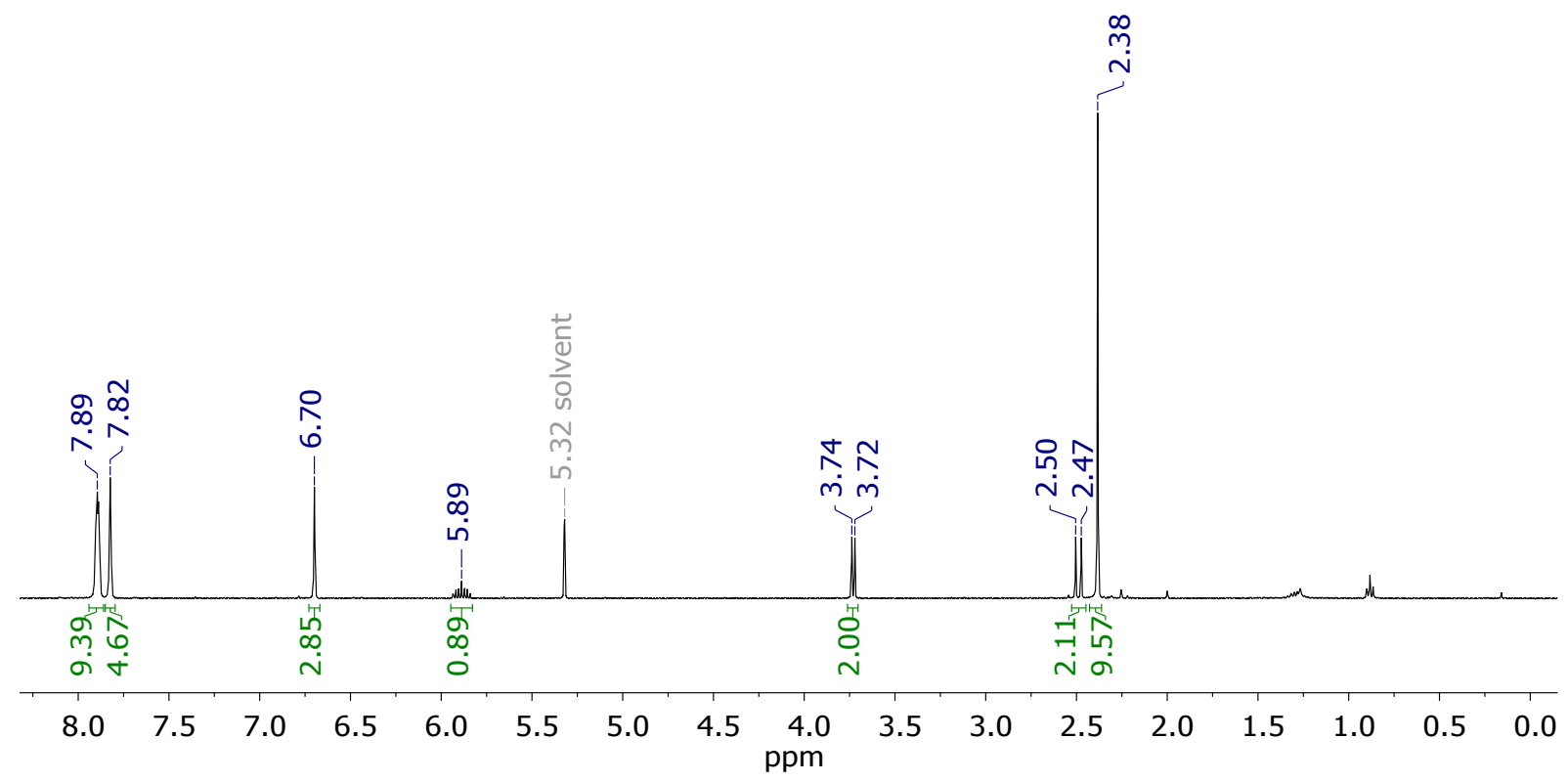

Figure S7. ${ }^{1} \mathrm{H}$ NMR spectrum of nickel(allyl)(mesitylene) tetrakis(3,5-bis(pentafluorosulfanyl)phenyl)borate, recorded in dichloromethane- $d_{2}$.

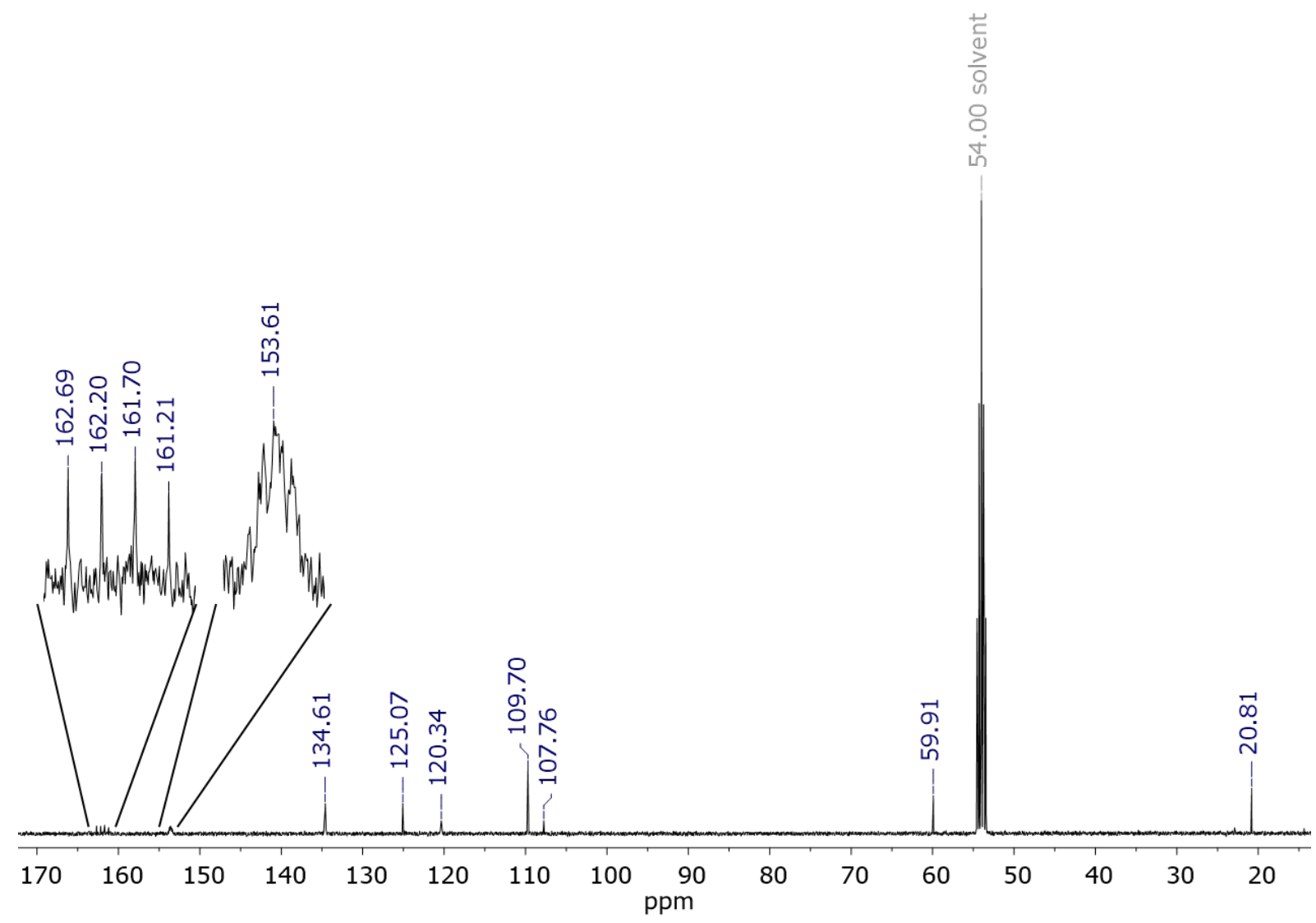

Figure S8. ${ }^{13} \mathrm{C}\left\{{ }^{1} \mathrm{H}\right\}$ NMR spectrum of nickel(allyl)(mesitylene) tetrakis(3,5-bis(pentafluorosulfanyl)phenyl)borate, recorded in dichloromethane- $d_{2}$ at $300 \mathrm{~K}$. 


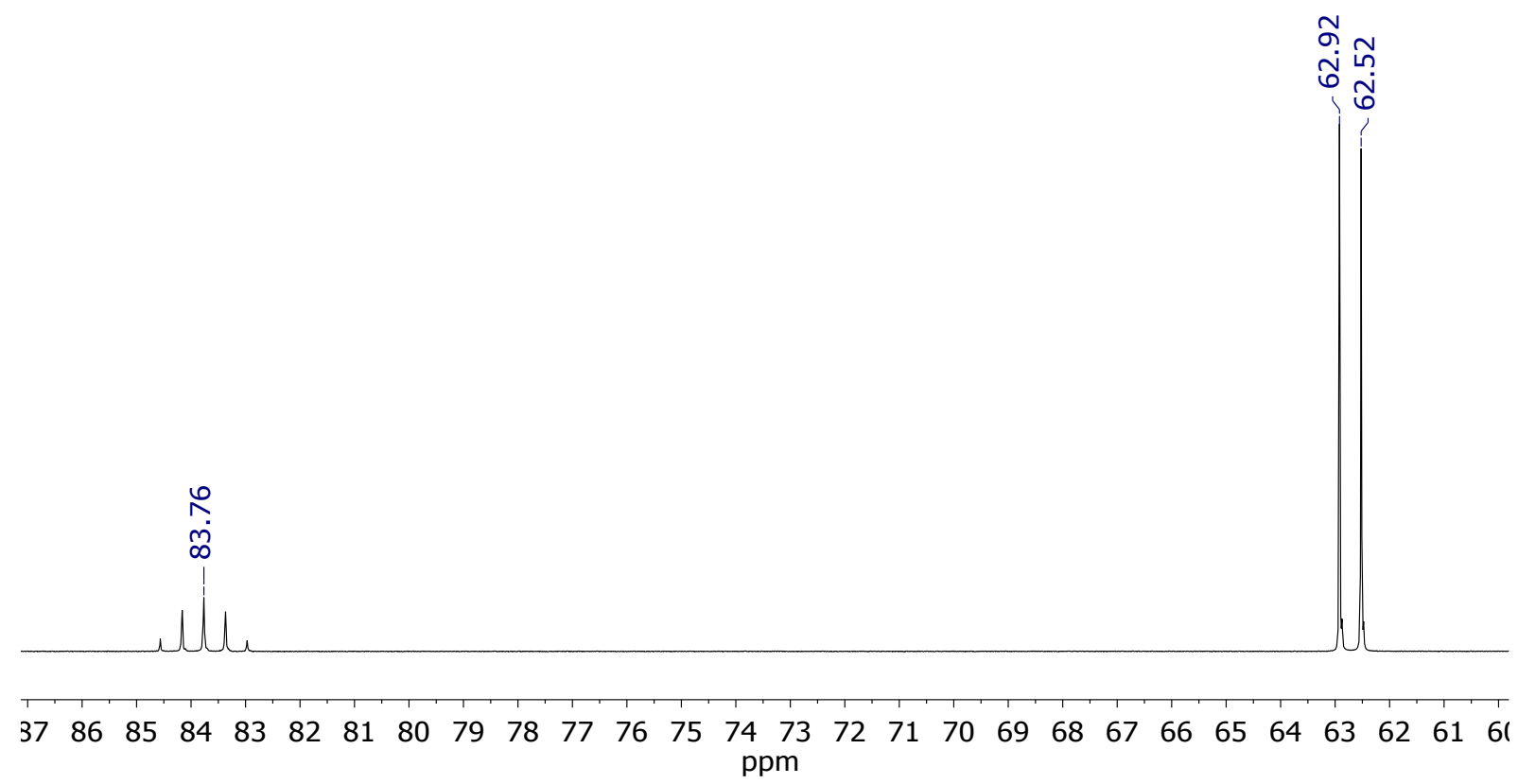

Figure S9. ${ }^{19} \mathrm{~F}$ NMR spectrum of nickel(allyl)(mesitylene) tetrakis(3,5-bis(pentafluorosulfanyl)phenyl)borate, recorded in dichloromethane- $d_{2}$ at $300 \mathrm{~K}$.

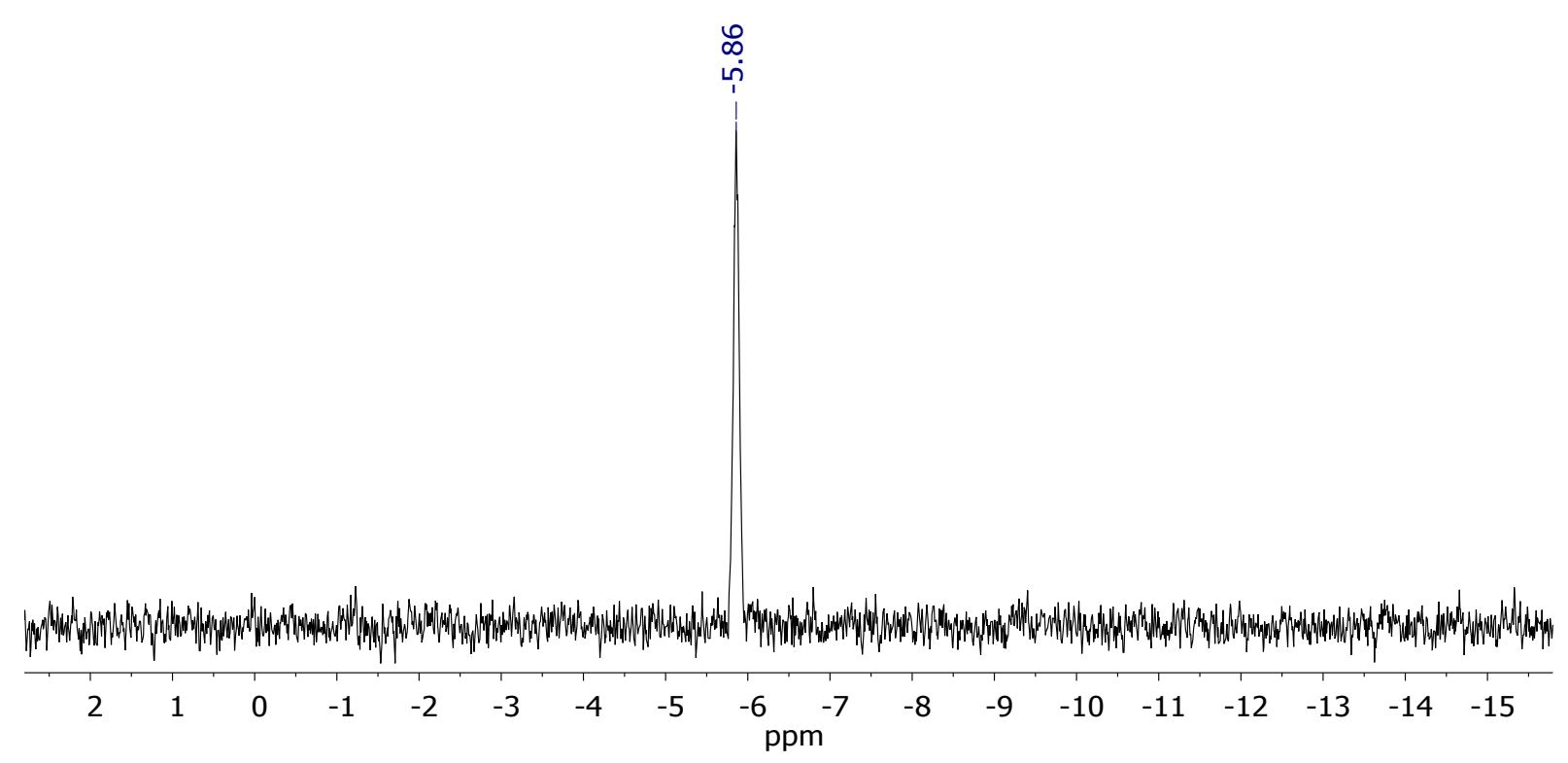

Figure S10. ${ }^{11} \mathrm{~B}$ NMR spectrum of nickel(allyl)(mesitylene) tetrakis(3,5-bis(pentafluorosulfanyl)phenyl)borate, recorded in dichloromethane- $d_{2}$ at $300 \mathrm{~K}$. 


\section{5 ${ }^{19}$ F NMR Spectra of Experiments with Highly Reactive Transmetalation Reagents}

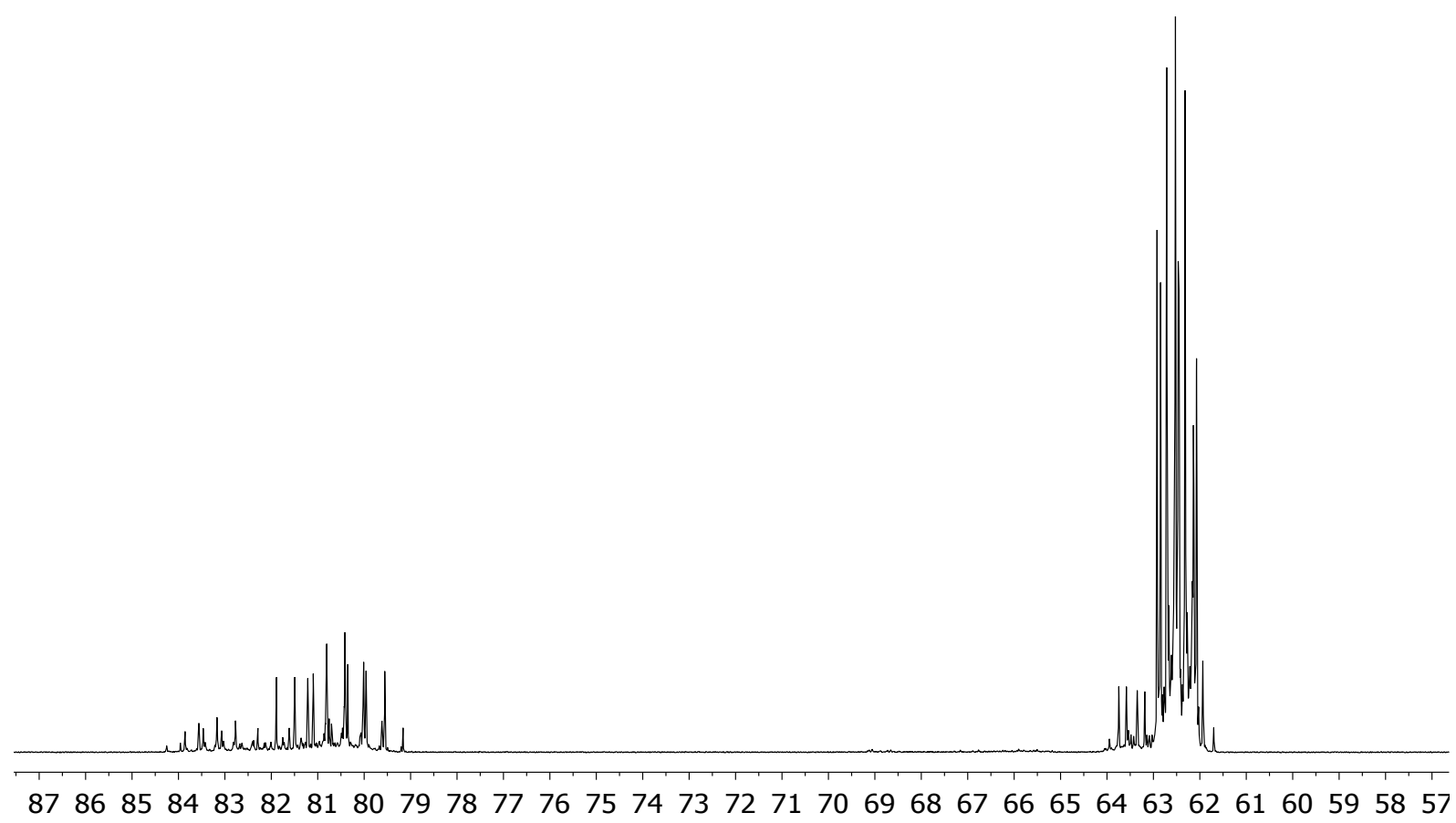

ppm

Figure S11. A representative ${ }^{19} \mathrm{~F}$ NMR spectrum of the high chemical shift region from an experiment with highly reactive transmetalation agents. Here, 1-bromo-3,5-bis(pentafluorosulfanyl)benzene was treated with a $1 \mathrm{M}$ solution of ${ }^{i} \mathrm{PrMgCl} \cdot \mathrm{LiCl}$ in THF. This spectrum was recorded in acetone- $d_{6}$ at $300 \mathrm{~K}$

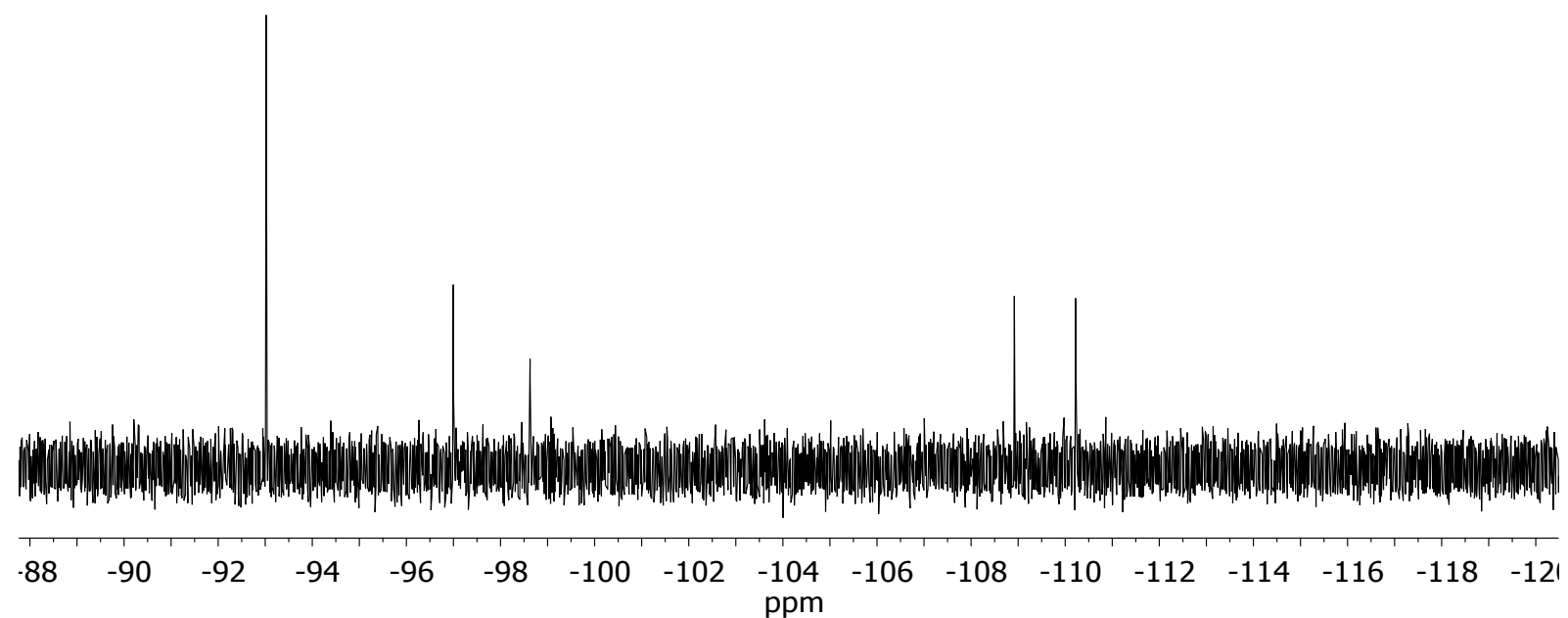

Figure S12. A representative ${ }^{19} \mathrm{~F}$ NMR spectrum of the low chemical shift region from an experiment with higher reactive transmetalation agents. Here, 1-bromo-3,5-bis(pentafluorosulfanyl)benzene was treated with a 1M solution of ${ }^{i} \mathrm{PrMgCl} \cdot \mathrm{LiCl}$ in THF. The spectrum was recorded in acetone- $d_{6}$ at $300 \mathrm{~K}$ 


\section{Single Crystal Structures}

\section{1 $\mathrm{Na}(\text { thf })_{5}-\mu-\mathrm{O}-(\mathrm{HO})-\mathrm{B}\left(\mathrm{Ar}^{\mathrm{SF5}}\right)_{3}$}

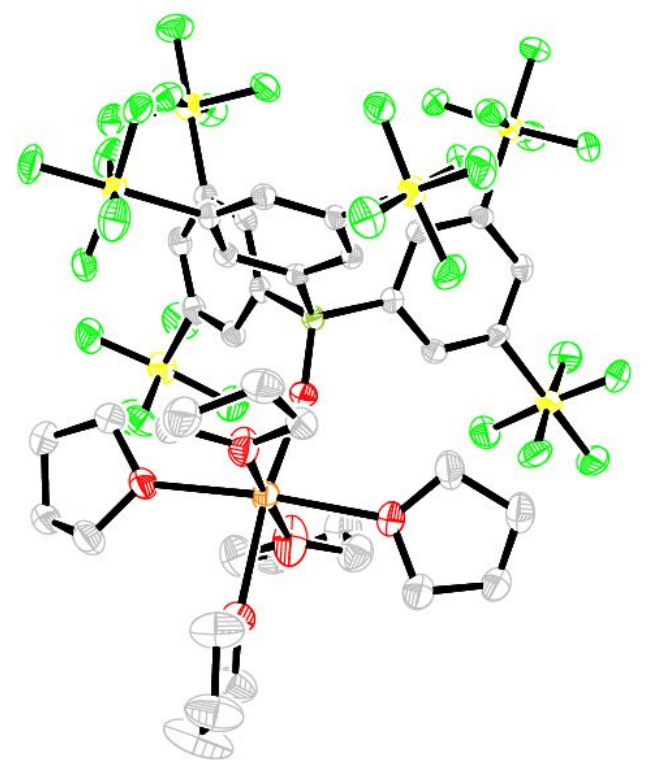

Figure S13. ORTEP diagram of the single crystal structure resolving the connectivity of $\left[\mathrm{Na}(\mathrm{thf})_{5}-\mu-\mathrm{O}-(\mathrm{HO})-\mathrm{B}\left(\mathrm{Ar}{ }^{\mathrm{SF} 5}\right)_{3}\right]$. Hydrogen atoms were omitted for clarity. Crystals suitable for SC-XRD were obtained by layering a THF solution with pentane. Bright green: fluorine, yellow: sulfur, gray: carbon, pale green: boron, red: oxygen, orange: sodium. 
Table S1: Crystal data and structure refinement for $\left[\mathrm{Na}(\mathrm{thf})_{5}-\mu-\mathrm{O}-(\mathrm{HO})-\mathrm{B}\left(\mathrm{Ar}^{\mathrm{SF} 5}\right)_{3}\right]$.

\begin{tabular}{|c|c|}
\hline CCDC no. & 1916541 \\
\hline Empirical formula & $\mathrm{C}_{38} \mathrm{H}_{50} \mathrm{BF}_{30} \mathrm{NaO}_{6} \mathrm{~S}_{6}$ \\
\hline Formula weight & 1398.94 \\
\hline Temperature/K & $100(2)$ \\
\hline Crystal system & monoclinic \\
\hline Space group & $\mathrm{P} 2{ }_{1} / \mathrm{n}$ \\
\hline$a / \AA$ & $12.6664(15)$ \\
\hline$b / \AA$ & $20.2764(18)$ \\
\hline$c / \AA$ & $21.143(3)$ \\
\hline$\alpha /^{\circ}$ & 90 \\
\hline$\beta /^{\circ}$ & $97.449(9)$ \\
\hline$\gamma /{ }^{\circ}$ & 90 \\
\hline Volume $/ \AA^{3}$ & $5384.4(10)$ \\
\hline$Z$ & 4 \\
\hline$\rho_{\text {calc }} \mathrm{g} / \mathrm{cm}^{3}$ & 1.726 \\
\hline$\mu / \mathrm{mm}^{-1}$ & 0.410 \\
\hline$F(000)$ & 2832.0 \\
\hline Crystal size $/ \mathrm{mm}^{3}$ & $0.45 \times 0.25 \times 0.2$ \\
\hline Radiation & $\operatorname{MoK}_{\alpha}(\lambda=0.71073)$ \\
\hline $2 \Theta$ range for data collection $/{ }^{\circ}$ & 3.99 to 53.608 \\
\hline Index ranges & $-16 \leq \mathrm{h} \leq 16,-25 \leq \mathrm{k} \leq 25,-26 \leq 1 \leq 26$ \\
\hline Reflections collected & 66113 \\
\hline Independent reflections & $11417\left[\mathrm{R}_{\mathrm{int}}=0.1041, \mathrm{R}_{\text {sigma }}=0.0588\right]$ \\
\hline Data/restraints/parameters & $11417 / 0 / 742$ \\
\hline Goodness-of-fit on $\mathrm{F}^{2}$ & 0.973 \\
\hline Final $R$ indexes $[\mathrm{I}>=2 \sigma(\mathrm{I})]$ & $R_{1}=0.0387, \mathrm{w} R_{2}=0.0862$ \\
\hline Final $R$ indexes [all data] & $R_{1}=0.0648, \mathrm{w} R_{2}=0.0930$ \\
\hline Largest diff. peak/hole / e $\AA^{-3}$ & $0.57 /-0.40$ \\
\hline
\end{tabular}




\subsection{Nickel(allyl)(mesitylene) Tetrakis(3,5-bis(pentafluoro- sulfanyl)phenyl)borate}

Note: 155 electrons were removed from data by Platon Squeeze routine for evaluation. The quality of data is still poor and disorder of one of two crystallographic independent nickel allyl mesitylene fragments further complicates improvements in the model. However, the connectivity and identity of the compound is established.

Table S2: Crystal data and structure refinement for nickel(allyl)(mesitylene) tetrakis(3,5-bis(pentafluorosulfanyl)phenyl)borate.

\begin{tabular}{ll}
\hline CCDC no. & 1916542 \\
\hline Empirical formula & $\mathrm{C}_{36} \mathrm{H}_{29} \mathrm{BF}_{40} \mathrm{~S}_{8} \mathrm{Ni}$ \\
Formula weight & 1546.58 \\
Temperature/K & 100.15 \\
Crystal system & triclinic \\
Space group & $\mathrm{P}-1$ \\
$a / \AA$ & $15.0727(6)$ \\
$b / \AA$ & $18.7542(7)$ \\
$c / \AA$ & $22.2073(9)$ \\
$\alpha /{ }^{\circ}$ & $97.725(3)$ \\
$\beta /{ }^{\circ}$ & $97.005(3)$ \\
$\gamma /{ }^{\circ}$ & $107.627(3)$ \\
Volume/ $\AA^{3}$ & $5839.8(4)$ \\
$Z(Z)$ & $4(2)$ \\
$\rho_{\text {calc }} /$ cm $^{3}$ & 1.759 \\
$\mu / \mathrm{mm}^{-1}$ & 0.776 \\
$F(000)$ & 3060.0 \\
Crystal size/mm & \\
Radiation & $0.45 \times 0.08 \times 0.04$ \\
$2 \Theta$ range for data collection ${ }^{\circ}$ & $\mathrm{MoK}_{\alpha}(\lambda=0.71073)$ \\
Index ranges & 2.706 to 52.466 \\
Reflections collected & $-18 \leq \mathrm{h} \leq 18,-23 \leq \mathrm{k} \leq 23,-27 \leq 1 \leq 27$ \\
Independent reflections & 81827 \\
Data/restraints/parameters & $23387\left(R_{\text {int }}=0.0820, R_{\text {sigma }}=0.0774\right)$ \\
Goodness-of-fit on $\mathrm{F}^{2}$ & $23340 / 0 / 1538$ \\
Final $R$ indexes $[\mathrm{I}>=2 \sigma(\mathrm{I})]$ & 1.041 \\
Final $R$ indexes $[$ all data $]$ & $R_{1}=0.0971, \mathrm{w} R^{2}=0.2598$ \\
Largest diff. peak/hole / e $\AA^{-3}$ & $R_{1}=0.1381, \mathrm{w} R^{2}=0.2842$ \\
\hline
\end{tabular}




\section{Polymers}

\subsection{General Procedure for Butadiene Polymerization}

A Schlenk flask sealed with a rubber septum was charged with the desired amount of catalyst and with $20 \mathrm{~mL}$ of heptane. The catalyst dispersion was treated briefly in an ultrasonic bath and then stirred for 5 minutes. The polymerization was initiated by exchanging the nitrogen atmosphere by a butadiene atmosphere (butadiene pressure: 1.05 bar). The experiment was allowed to run at RT for 128 minutes prior to termination via addition of $1 \mathrm{~mL}^{\text {of }} \mathrm{NEt}_{3}$. The residual butadiene was removed under reduced pressure and the polymer was precipitated by adding the reaction mixture carefully to a solution of $50 \mathrm{mg}$ of 2,6-di-tert-butyl-4-methylphenol in $600 \mathrm{~mL}$ of $\mathrm{MeOH}$. The mixture was stirred for 75 minutes. The liquid phase was removed and the isolated polymer was stirred twice with further $500 \mathrm{~mL}$ of $\mathrm{MeOH}$ for 75 minutes each. The polymer was dried for a minimum of four days at $50{ }^{\circ} \mathrm{C}$ at reduced pressure.

\subsection{NMR Spectra of Polymers}

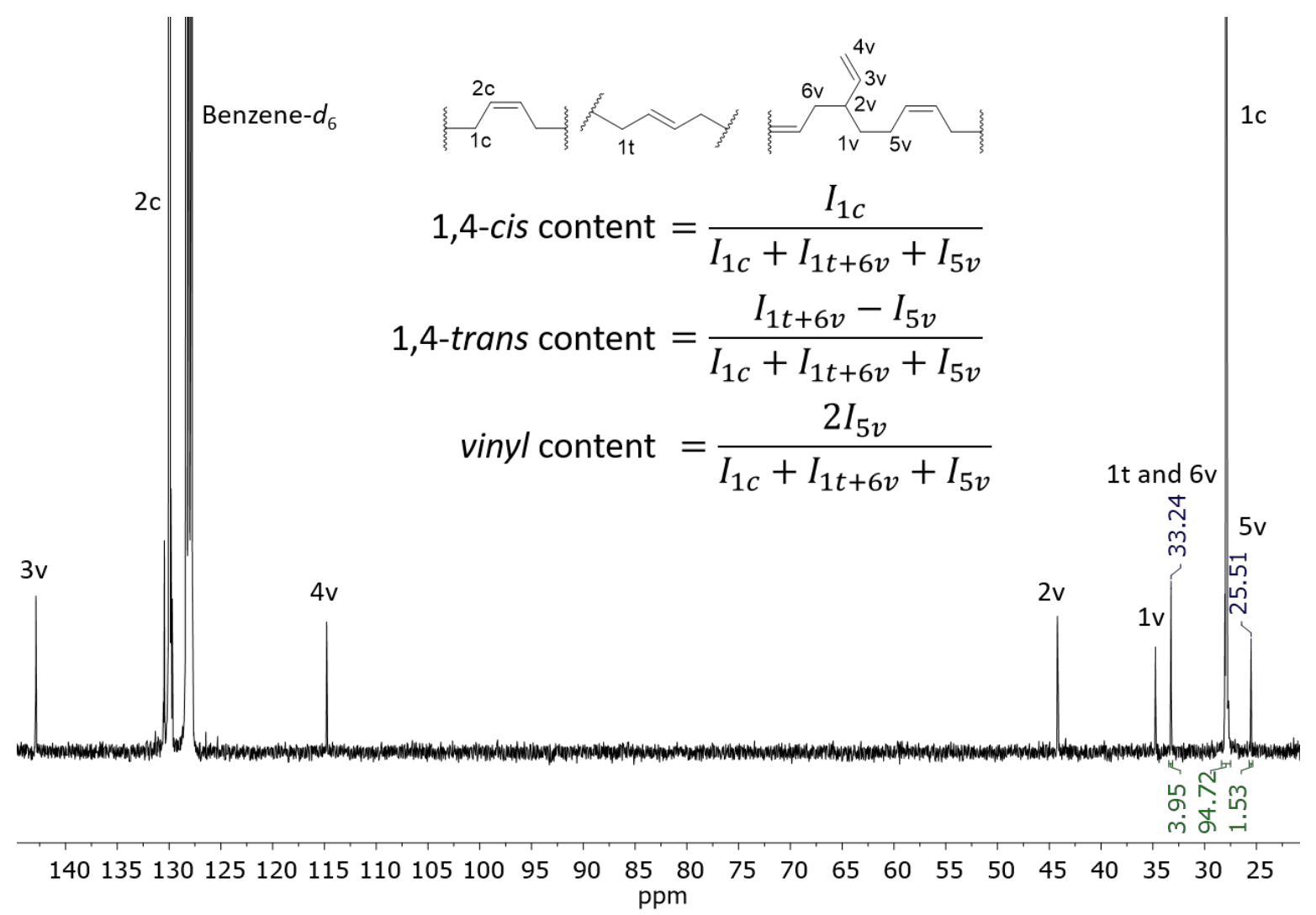

Figure S14: Inverse gated decoupled ${ }^{13} \mathrm{C}$ NMR spectrum of entry 1 (Table 1), recorded in benzene- $\mathrm{d}_{6}$ at $300 \mathrm{~K}$. 


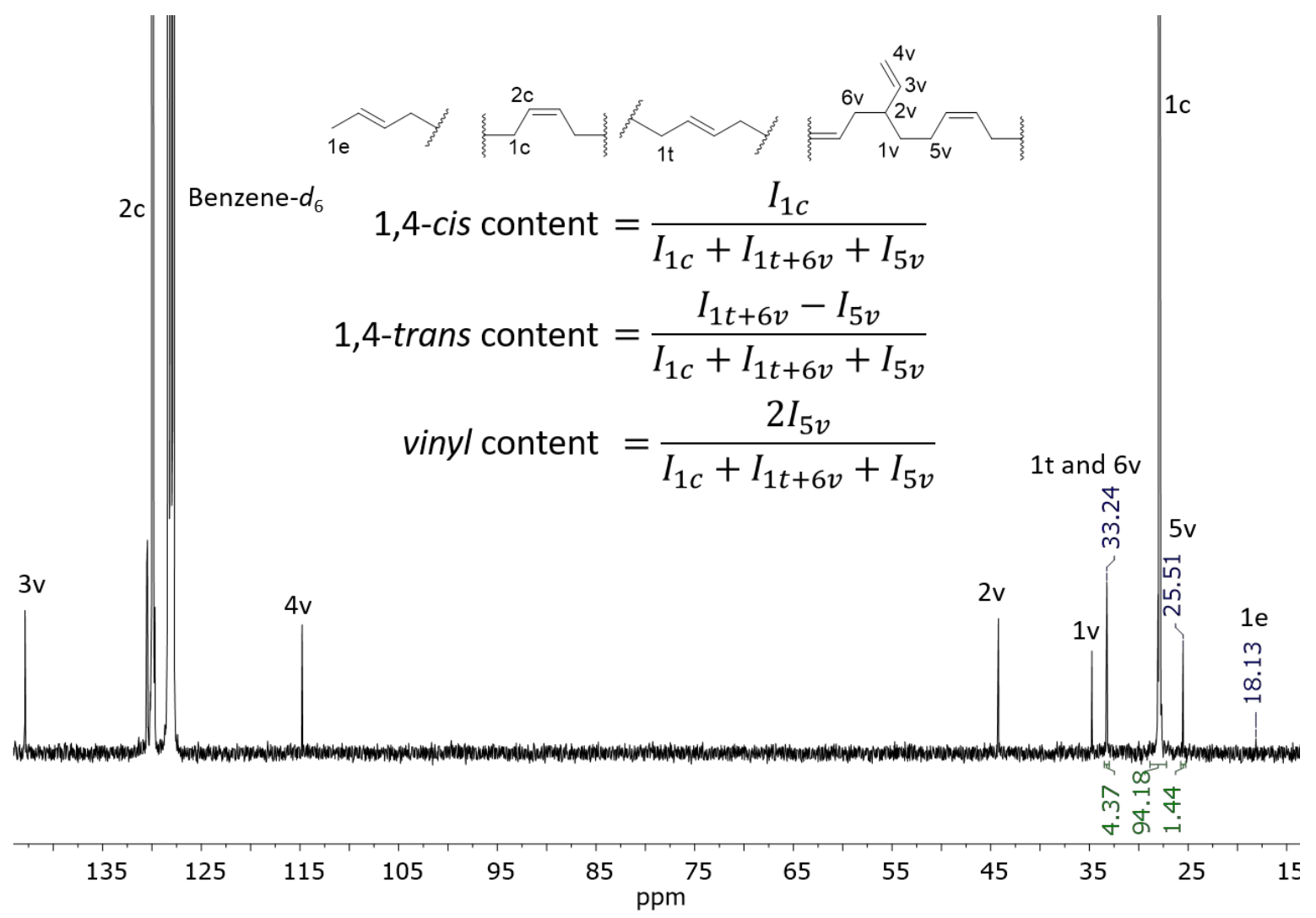

Figure S15: Inverse gated decoupled ${ }^{13} \mathrm{C}$ NMR spectrum of entry 2 (Table 1), recorded in benzene- $\mathrm{d}_{6}$ at $300 \mathrm{~K}$.

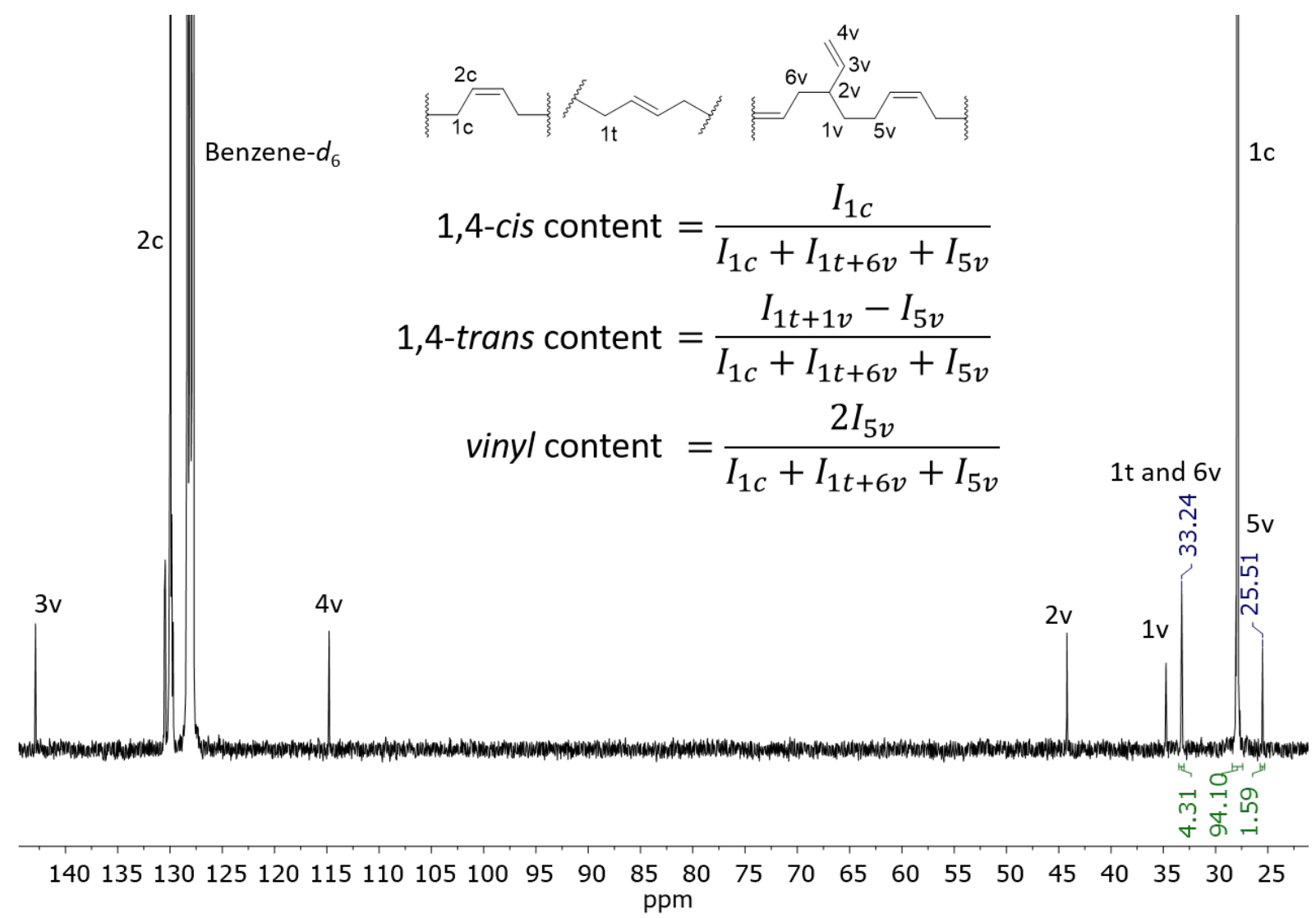

Figure S16: Inverse gated decoupled ${ }^{13} \mathrm{C}$ NMR spectrum of entry 3 (Table 1), recorded in benzene- $\mathrm{d}_{6}$ at $300 \mathrm{~K}$. 


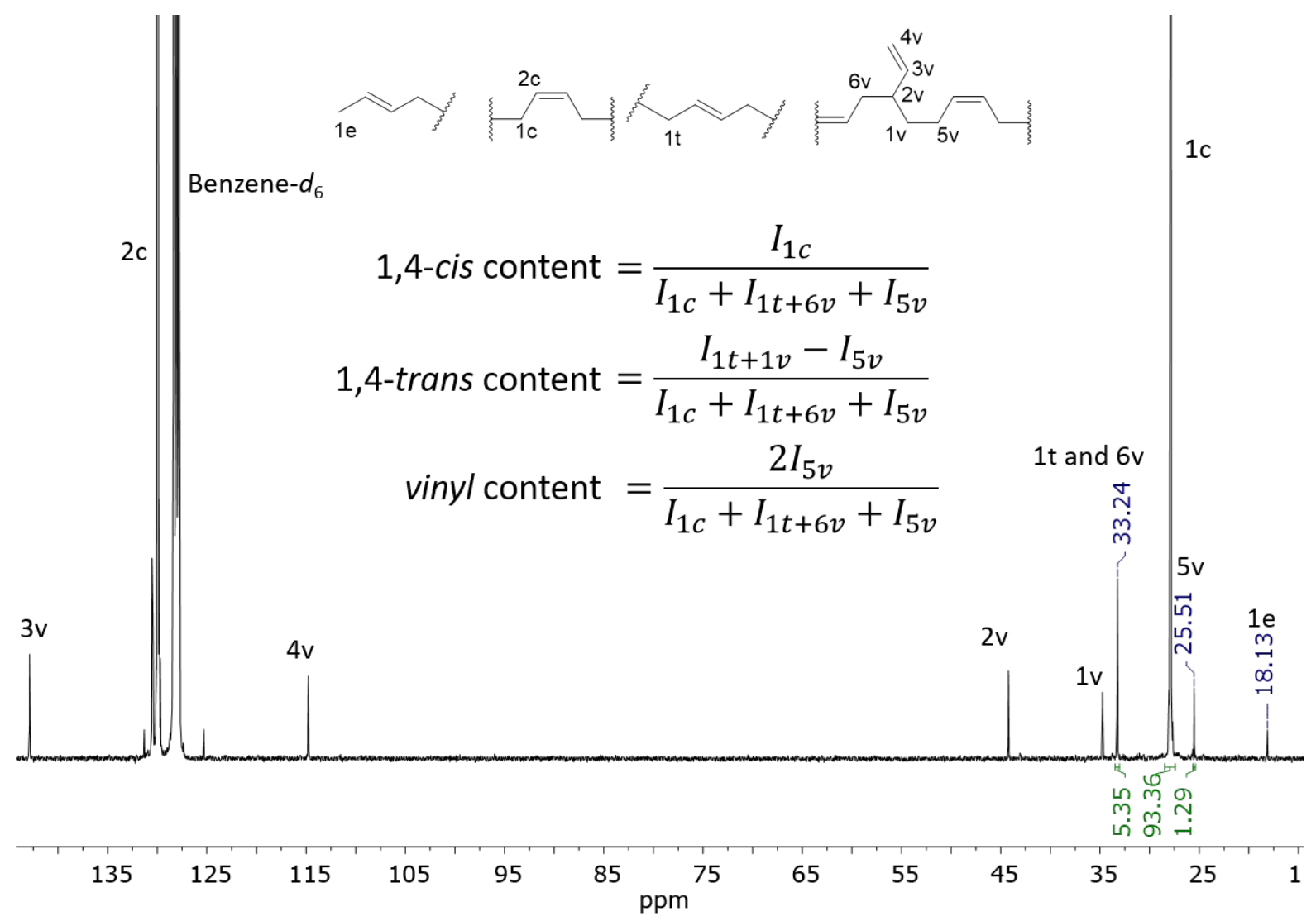

Figure S17: Inverse gated decoupled ${ }^{13} \mathrm{C}$ NMR spectrum of entry 4 (Table 1), recorded in benzene- $\mathrm{d}_{6}$ at $300 \mathrm{~K}$.

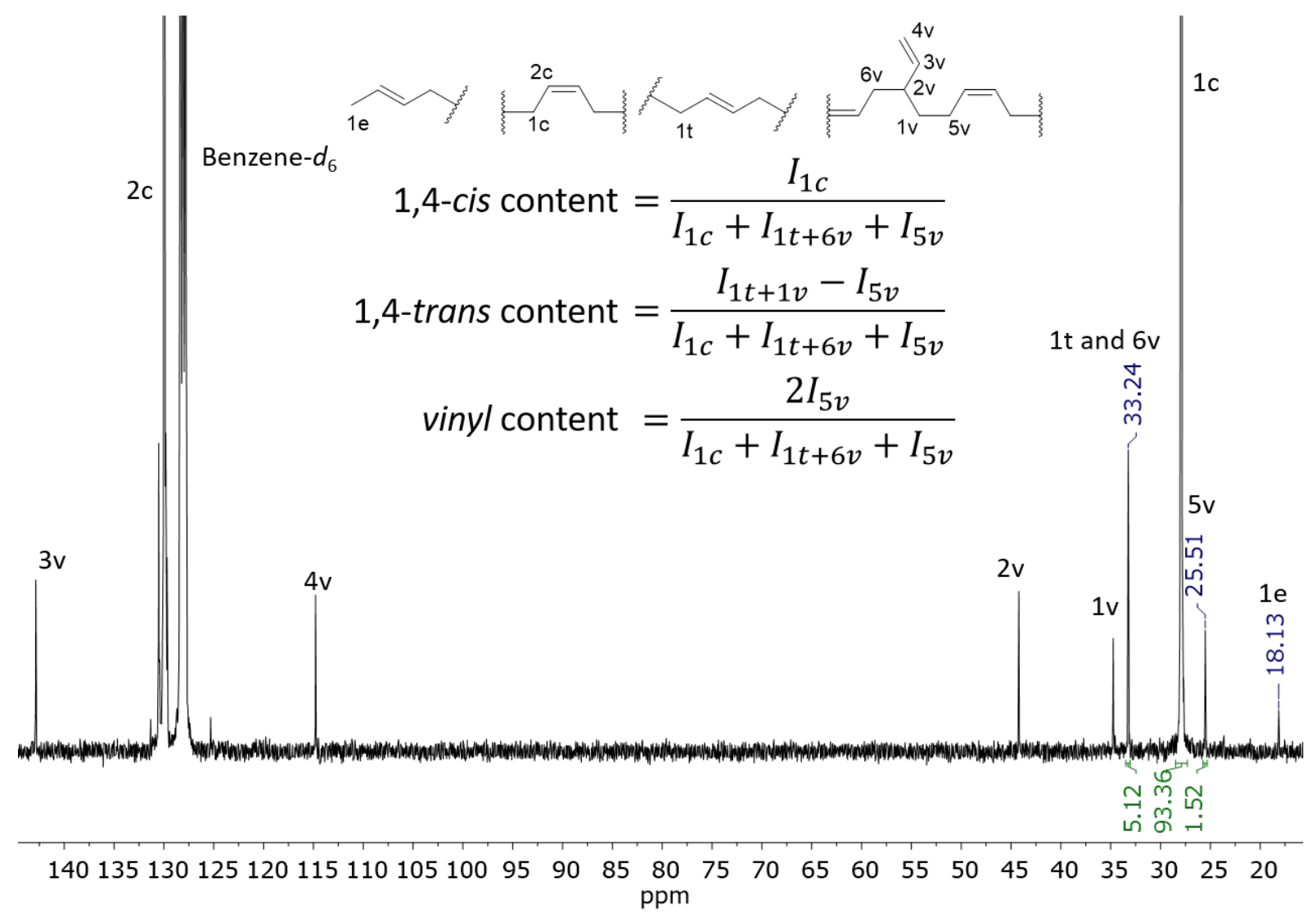

Figure S18: Inverse gated decoupled ${ }^{13} \mathrm{C}$ NMR spectrum of entry 5 (Table 1), recorded in benzene- $\mathrm{d}_{6}$ at $300 \mathrm{~K}$. 


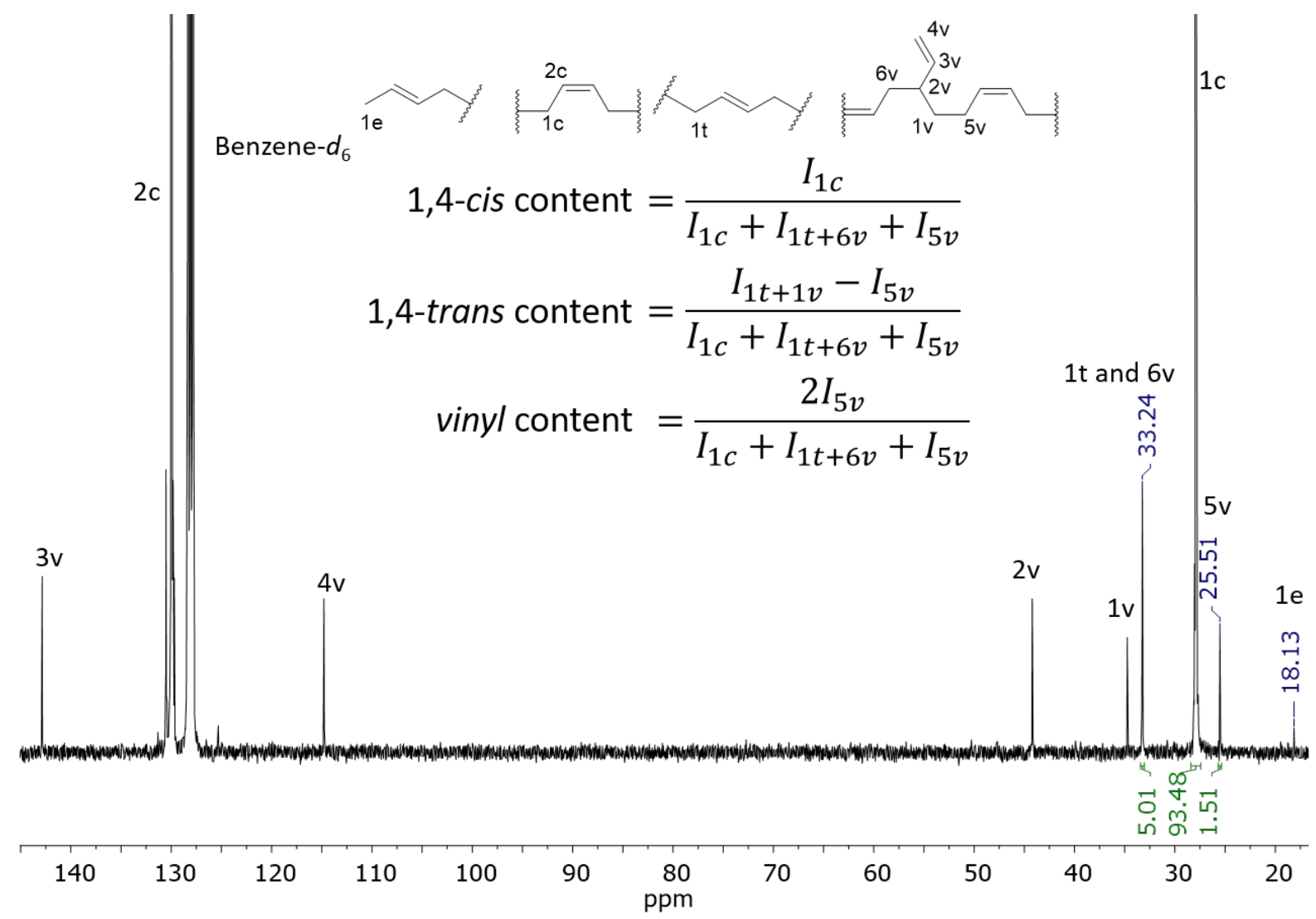

Figure S19: Inverse gated decoupled ${ }^{13} \mathrm{C}$ NMR spectrum of entry 6 (Table 1), recorded in benzene- $\mathrm{d}_{6}$ at $300 \mathrm{~K}$.

\subsection{GPC Traces of Polymers}

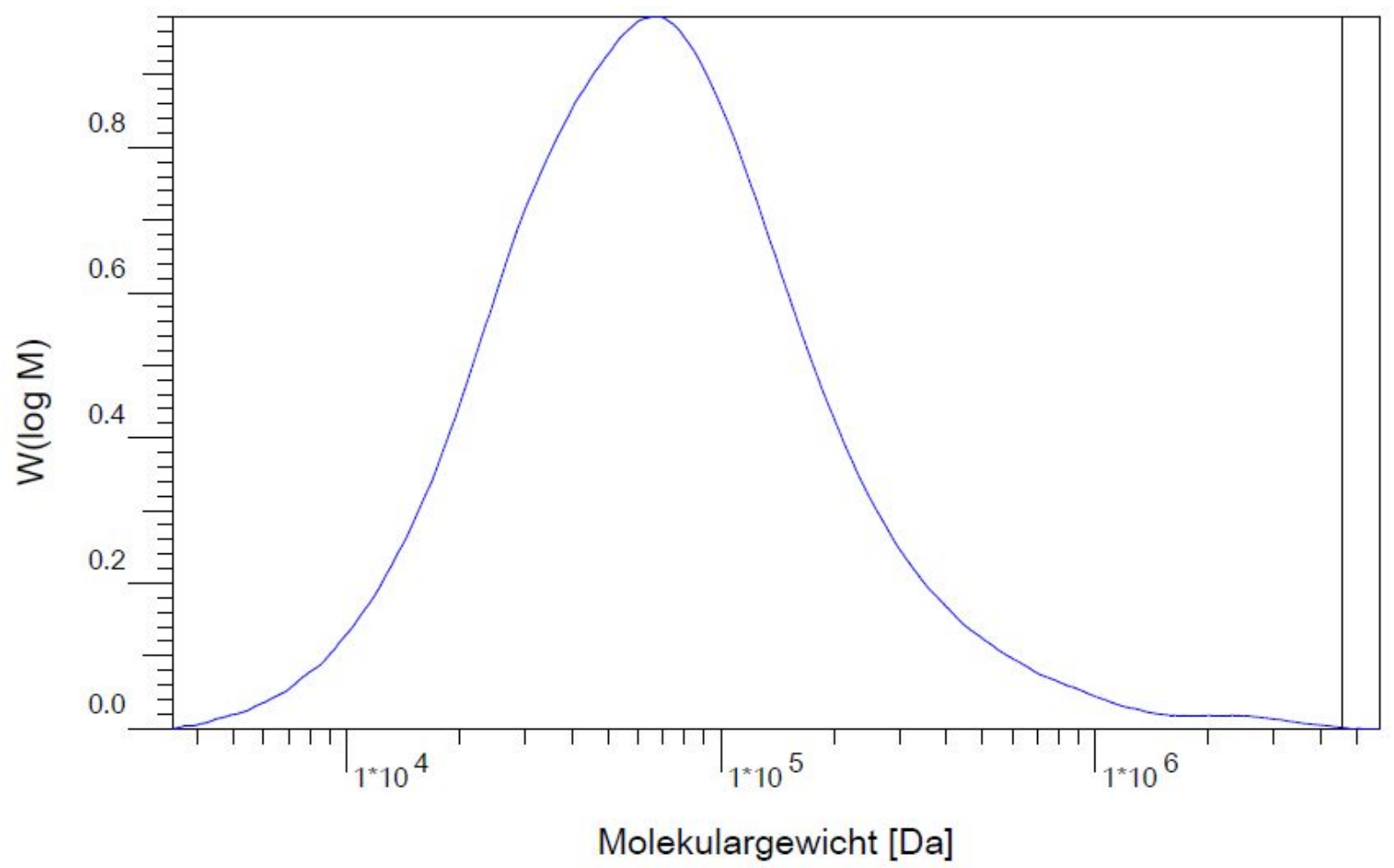

Figure S20: GPC trace of entry $\mathbf{1}$ (Table 1) measured in THF at $35^{\circ} \mathrm{C}$ against poly(styrene) standards with refractive index detection. 


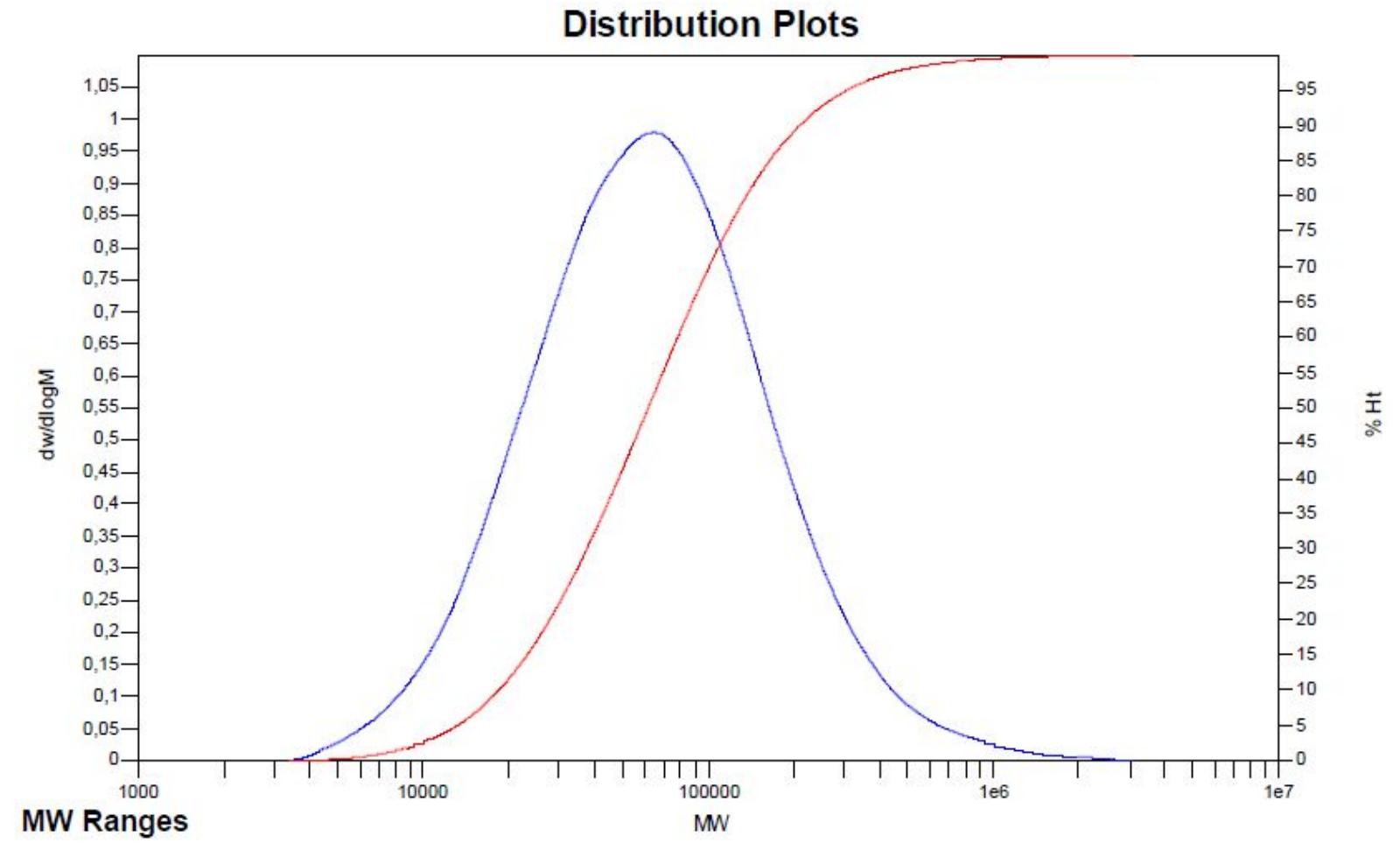

Figure S21: GPC trace of entry 2 (Table 1) measured in THF at $40{ }^{\circ} \mathrm{C}$ against poly(styrene) standards with refractive index detection.

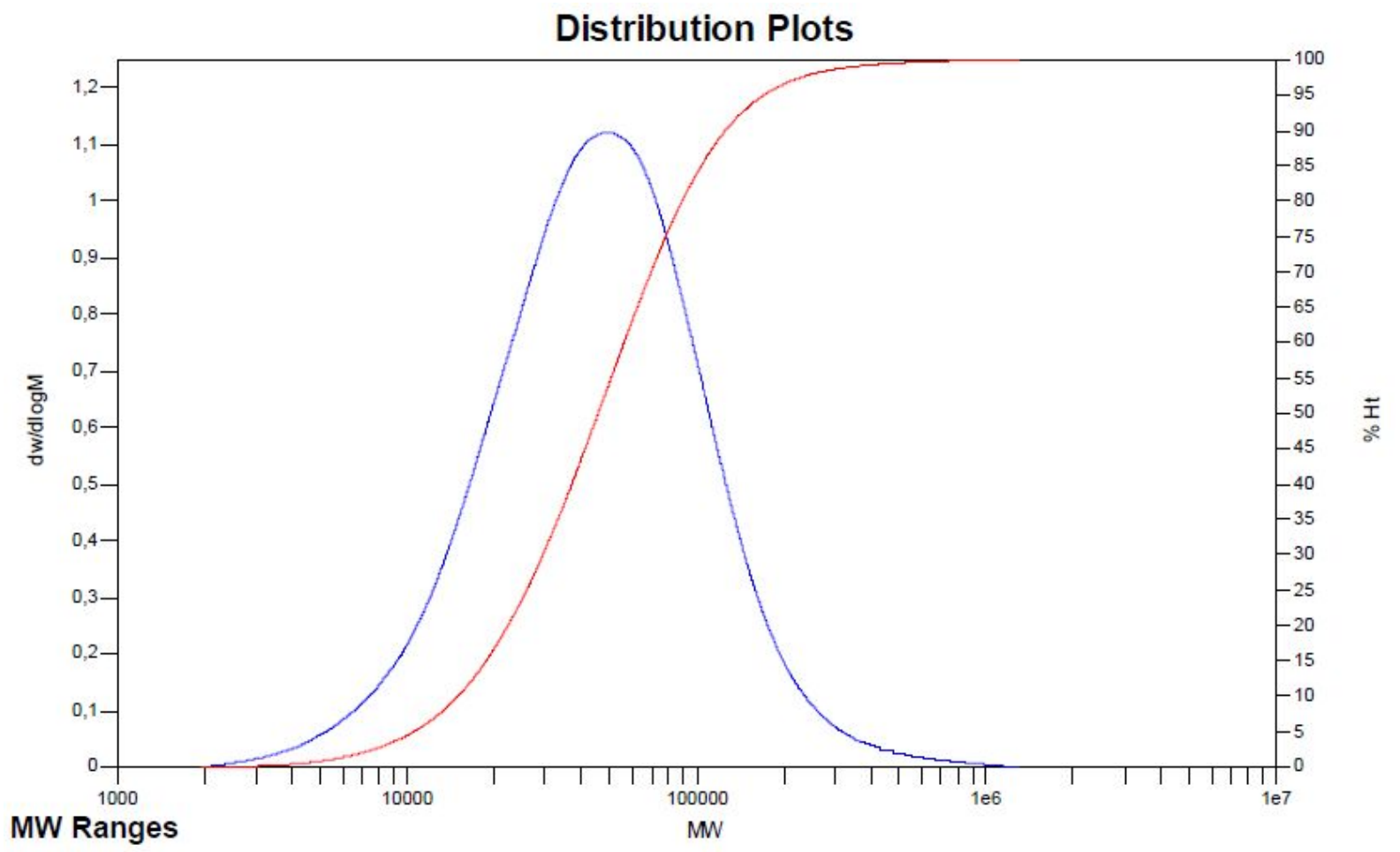

Figure S22: GPC trace of entry 3 (Table 1) measured in THF at $40{ }^{\circ} \mathrm{C}$ against poly(styrene) standards with refractive index detection. 


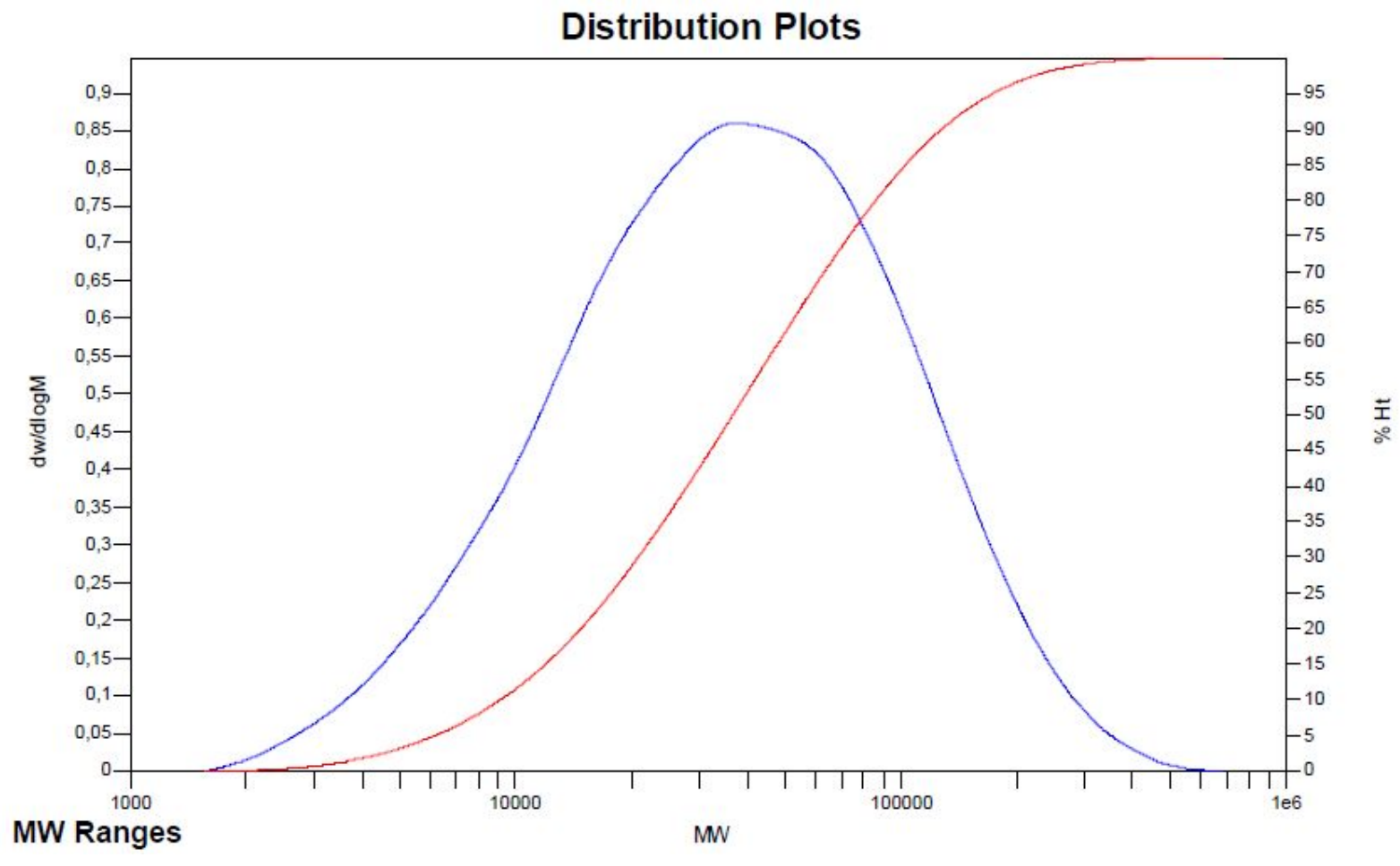

Figure S23: GPC trace of entry 4 (Table 1) measured in THF at $40{ }^{\circ} \mathrm{C}$ against poly(styrene) standards with refractive index detection.

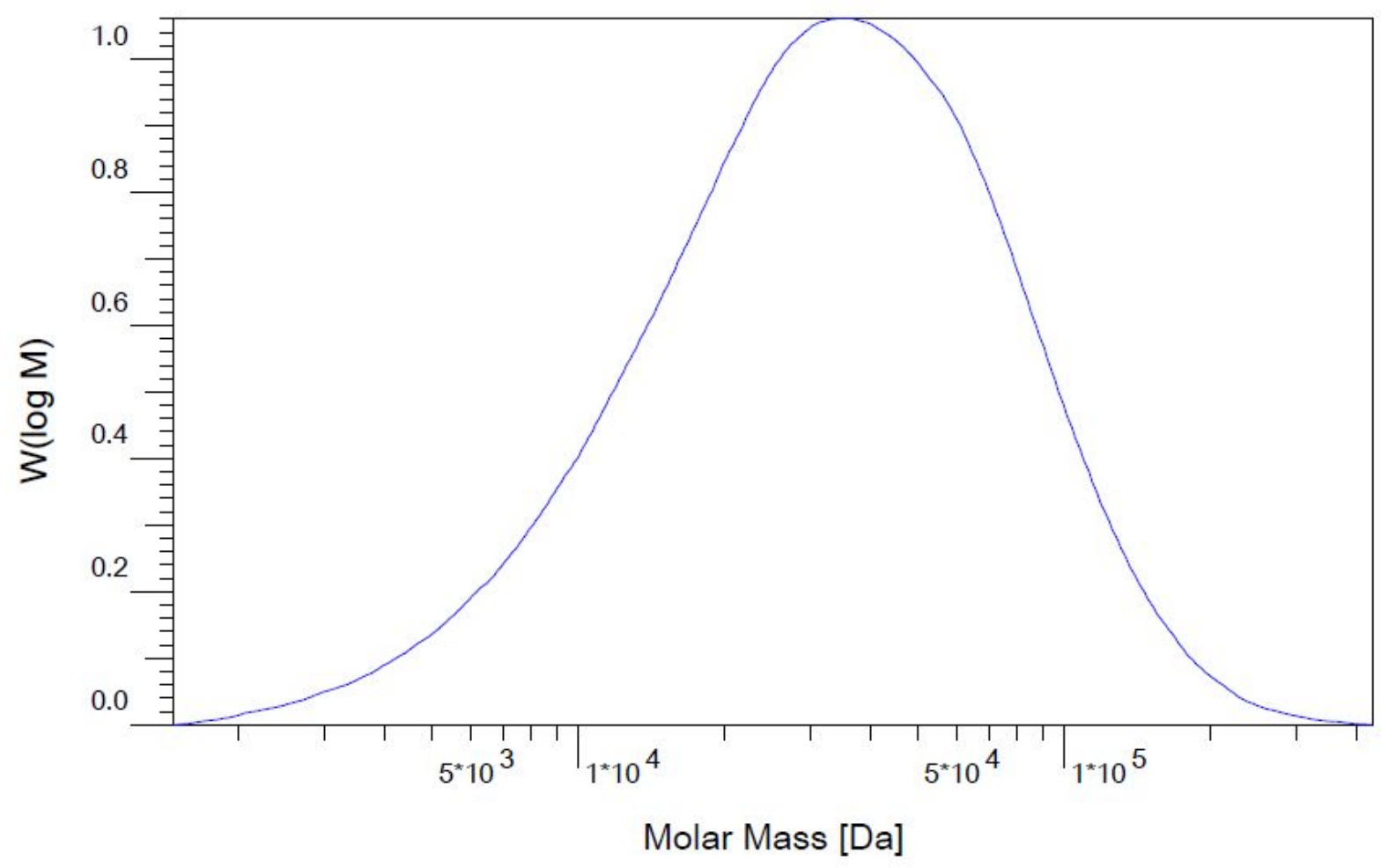

Figure S24: GPC trace of entry 5 (Table 1) measured in THF at $35^{\circ} \mathrm{C}$ against poly(styrene) standards with refractive index detection. 


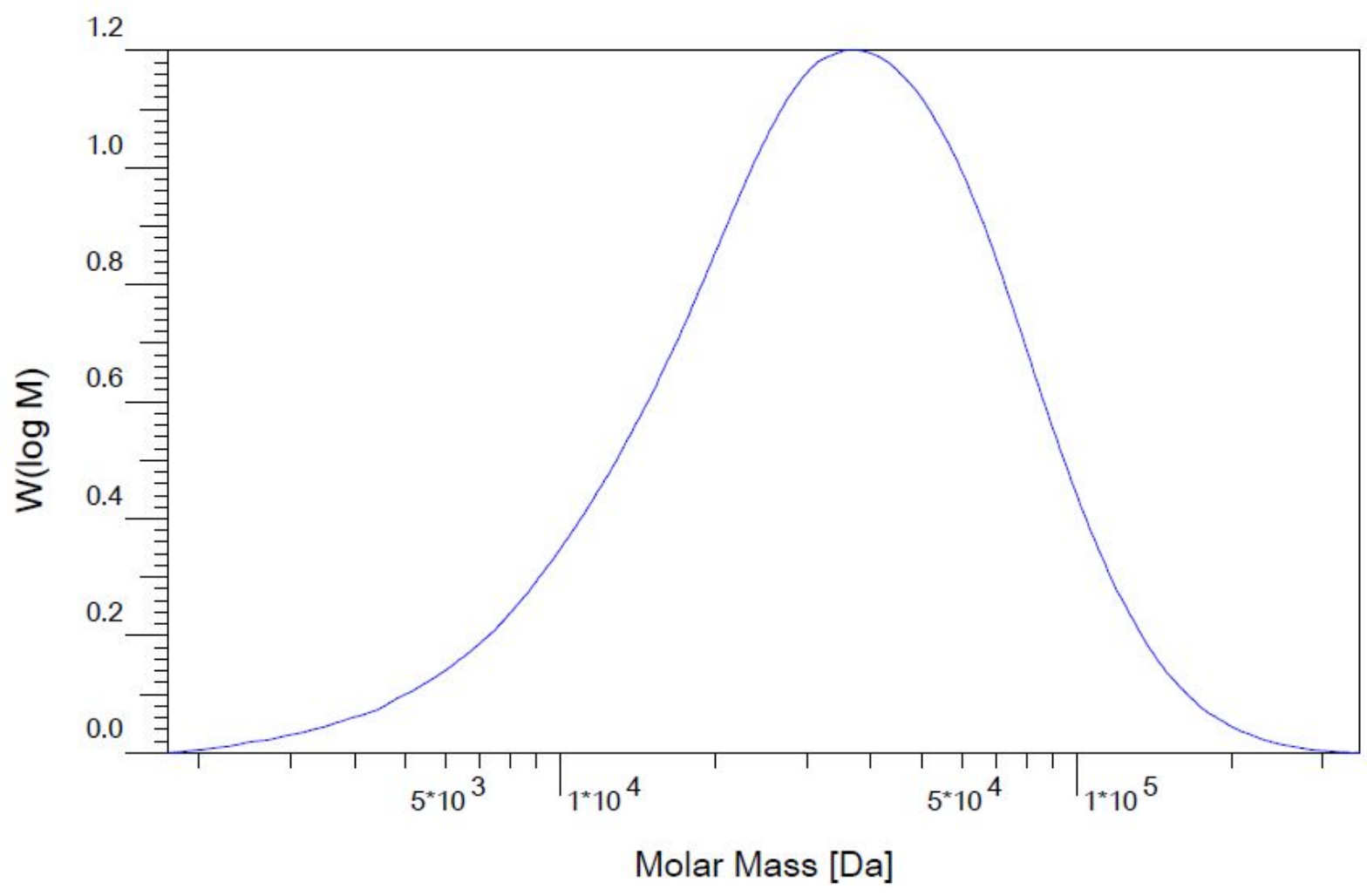

Figure S25: GPC trace of entry 6 (Table 1) measured in THF at $35^{\circ} \mathrm{C}$ against poly(styrene) standards with refractive index detection.

\subsection{DSC Data of Polymers}

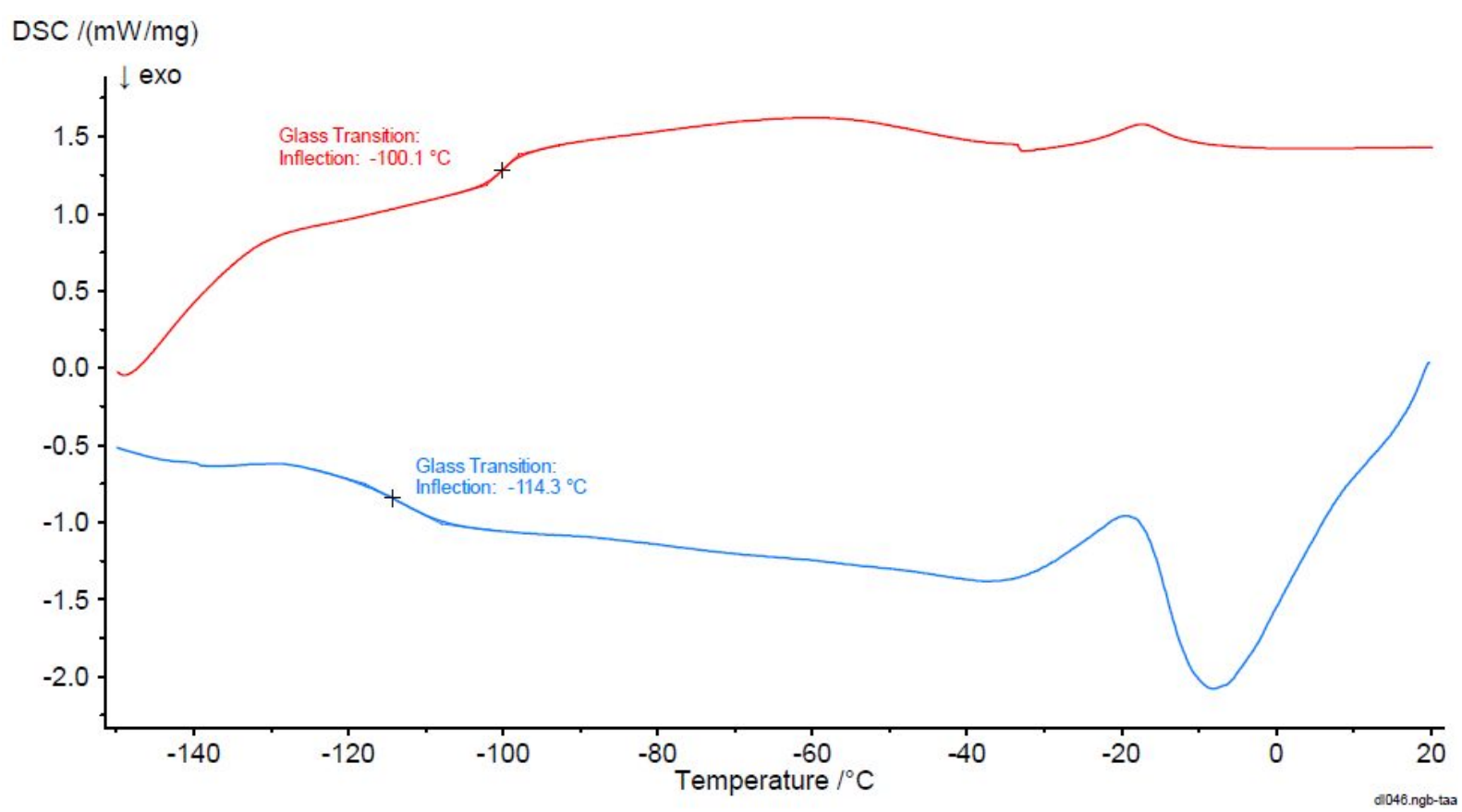

Figure S26: DSC data of entry 1 (Table 1). Applied heating and cooling rates: $30 \mathrm{~K} / \mathrm{min}$. Red: Heating curve, blue: Cooling curve. 


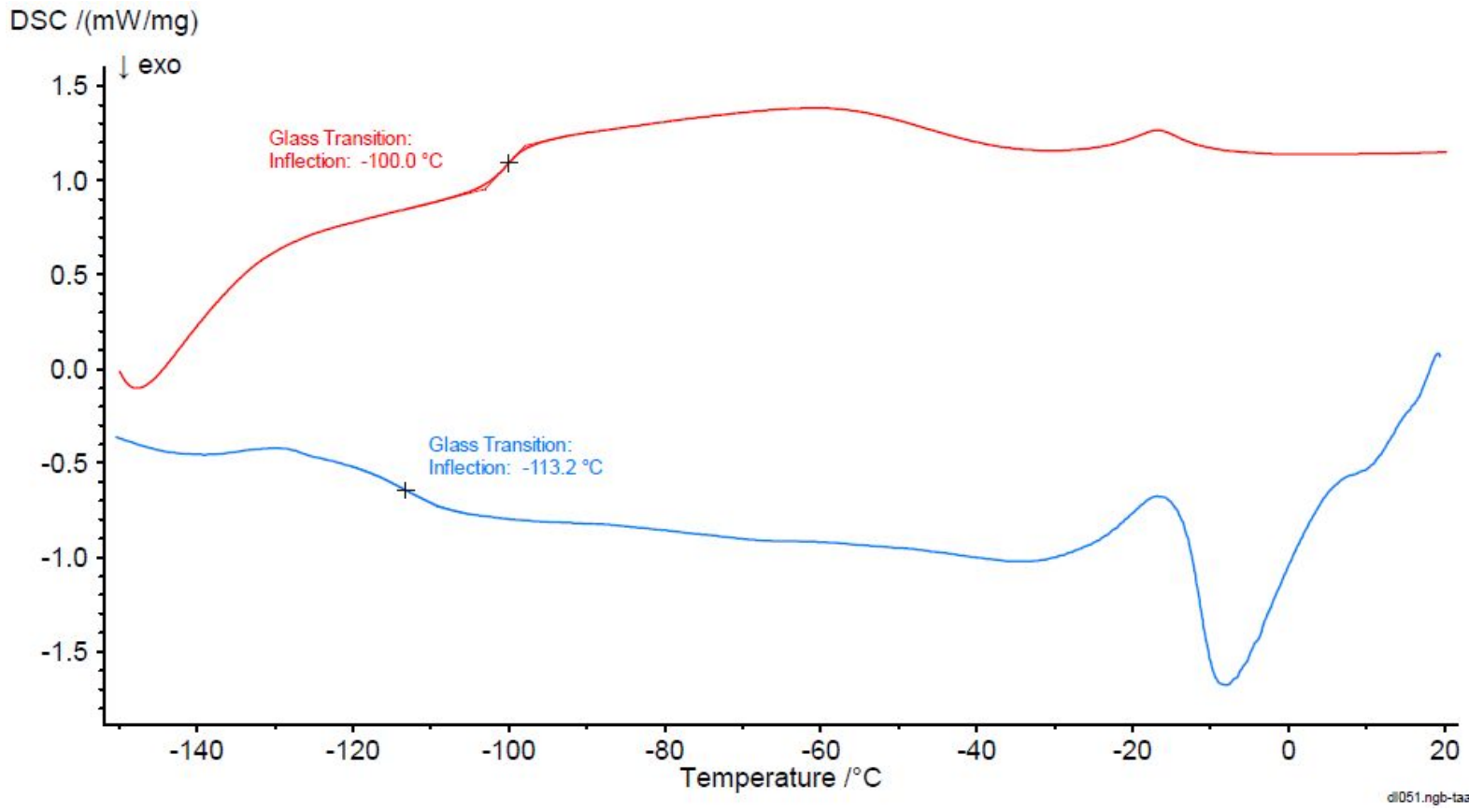

Figure 27: DSC data of entry 2 (Table 1). Applied heating and cooling rates: $30 \mathrm{~K} / \mathrm{min}$. Red: Heating curve, blue: Cooling curve.

$\mathrm{DSC} /(\mathrm{mW} / \mathrm{mg})$

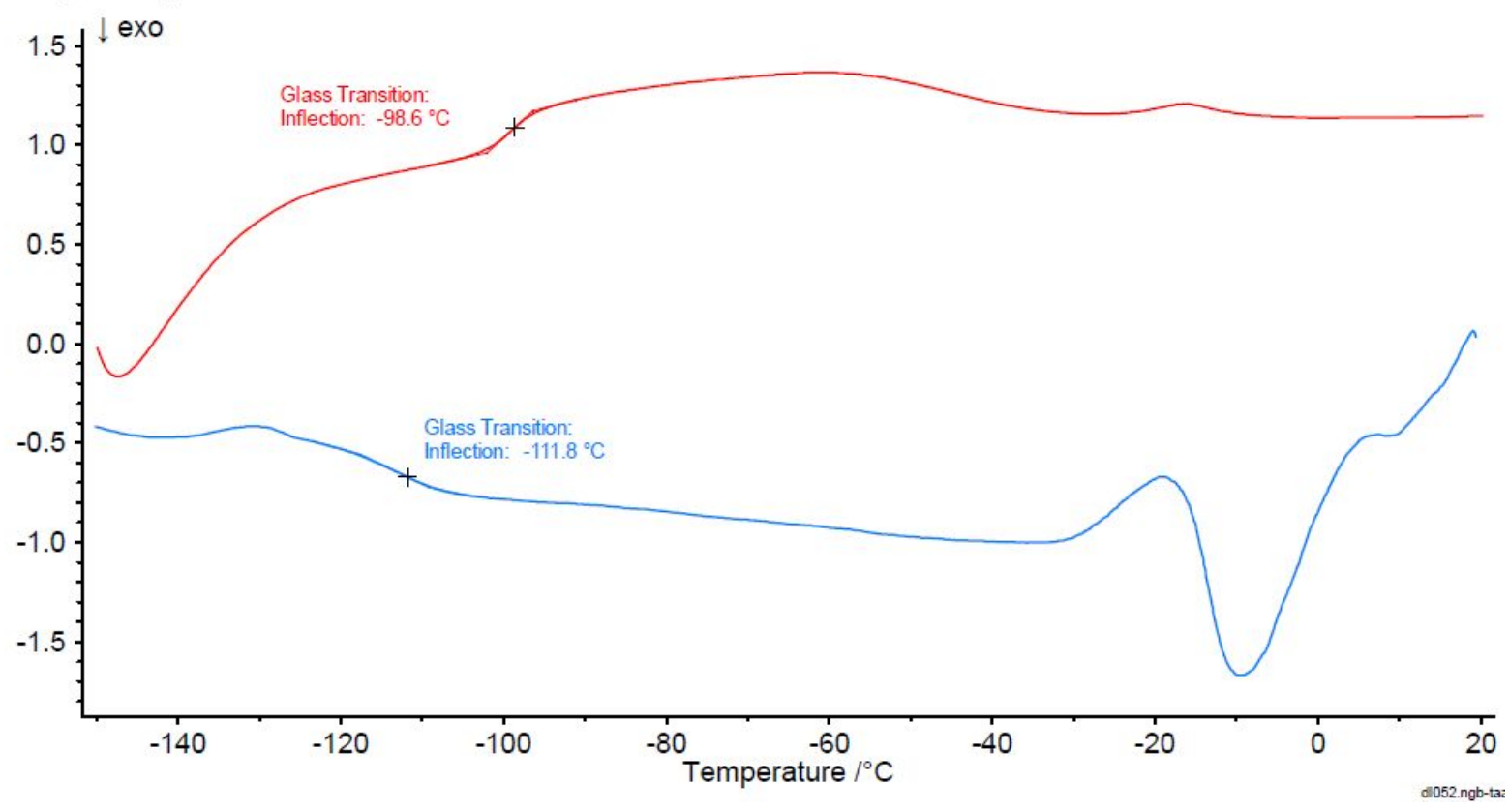

Figure S28: DSC data of entry 3 (Table 1). Applied heating and cooling rates: $30 \mathrm{~K} / \mathrm{min}$. Red: Heating curve, blue: Cooling curve. 


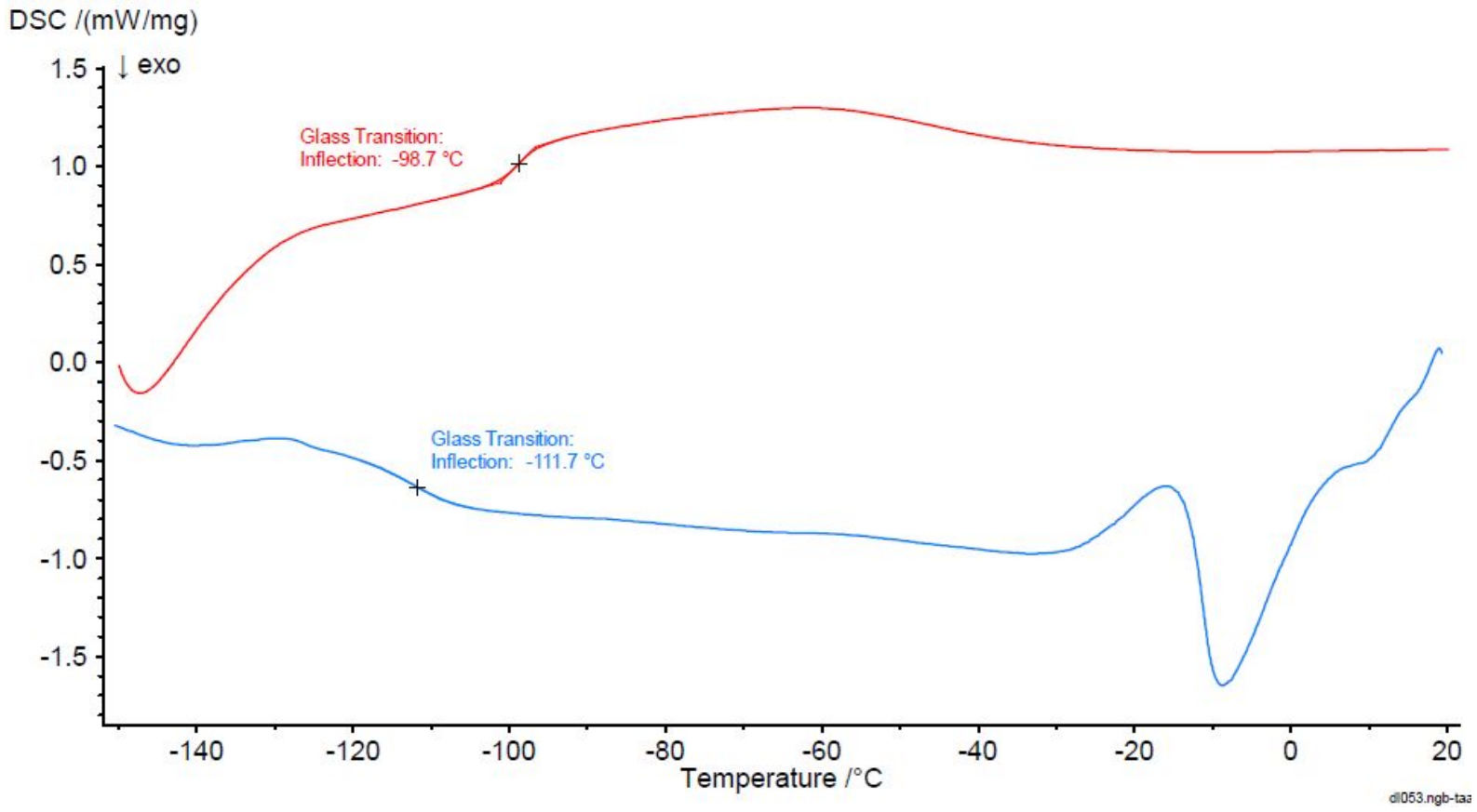

Figure S29: DSC data of entry 4 (Table 1). Applied heating and cooling rates: $30 \mathrm{~K} / \mathrm{min}$. Red: Heating curve, blue: Cooling curve.

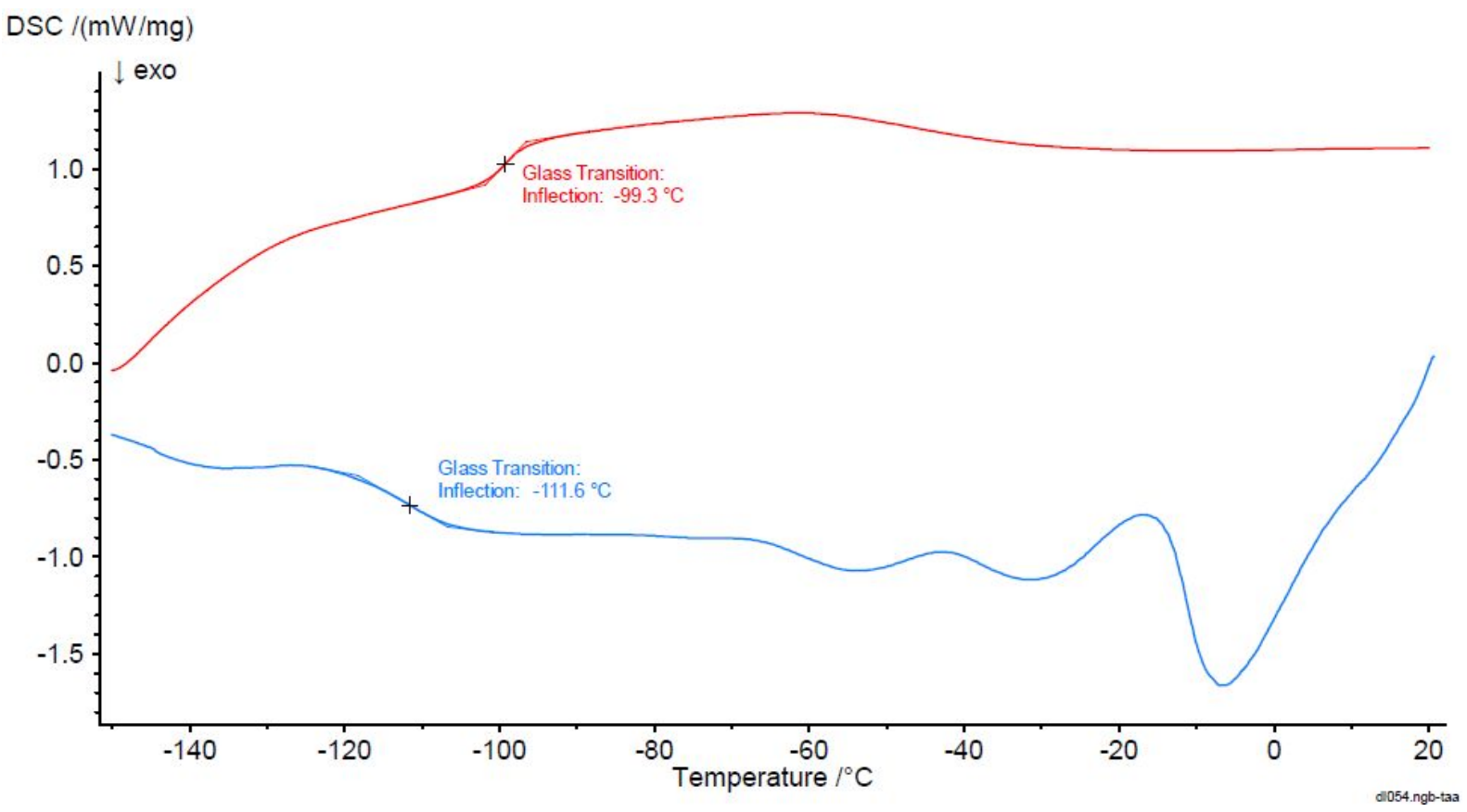

Figure S30: DSC data of entry 5 (Table 1). Applied heating and cooling rates: $30 \mathrm{~K} / \mathrm{min}$. Red: Heating curve, blue: Cooling curve. 


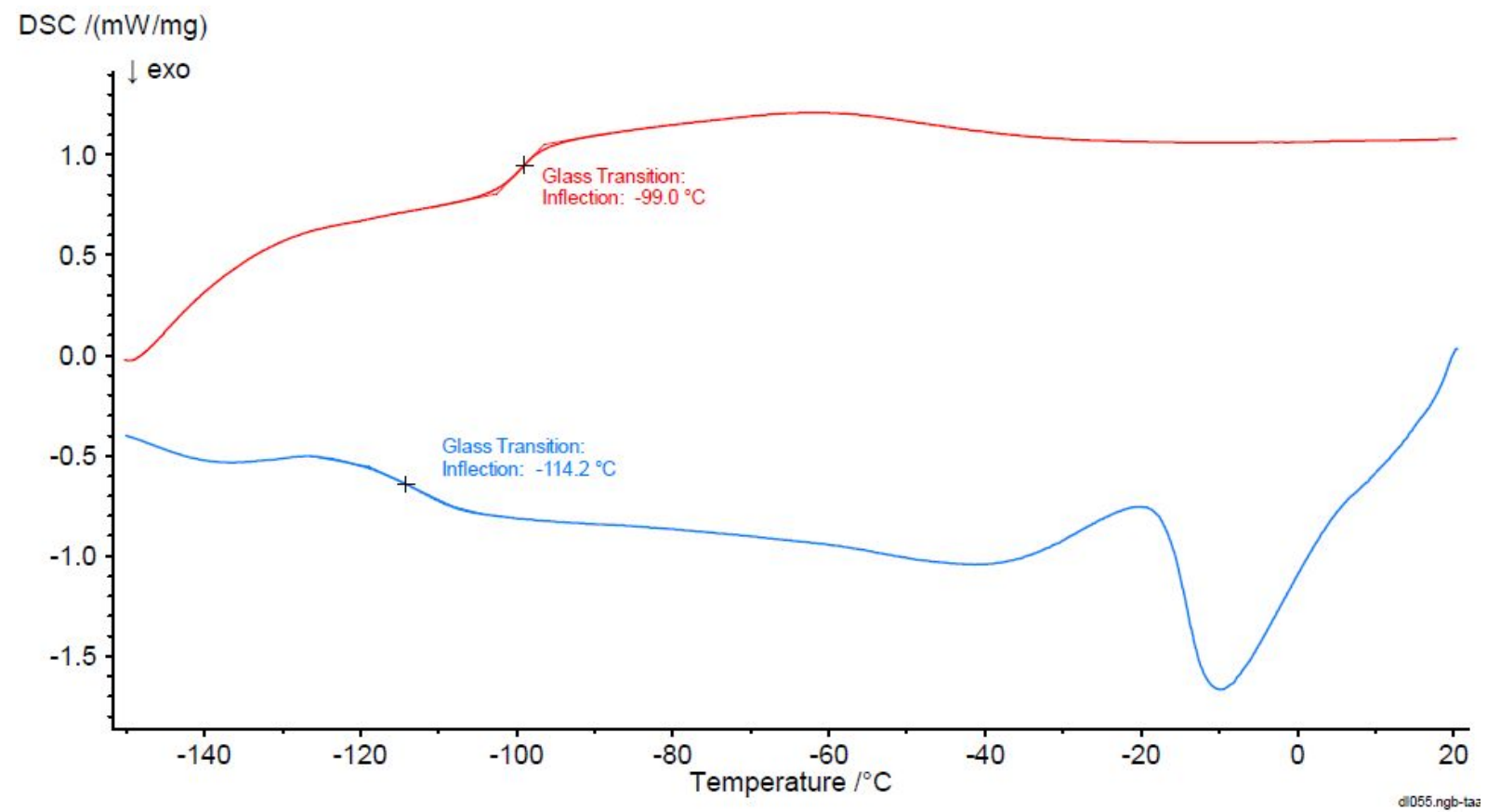

Figure S31: DSC data of entry 6 (Table 1). Applied heating and cooling rates: $30 \mathrm{~K} / \mathrm{min}$. Red: Heating curve, blue: Cooling curve. 


\section{DFT Calculations}

Table S3: Calculated orbital energies and Mulliken parameters of the fragments of [S-BArF] and [BArF].

\begin{tabular}{cccccccccc}
\hline Anion & MO & $\begin{array}{c}\text { Aryl 1 } \\
{[\%]}\end{array}$ & $\begin{array}{c}\text { Aryl 2 } \\
{[\%]}\end{array}$ & $\begin{array}{c}\text { Aryl 3 } \\
{[\%]}\end{array}$ & $\begin{array}{c}\text { Aryl 4 } \\
{[\%]}\end{array}$ & $\begin{array}{c}\Sigma(\text { Aryl }) \\
{[\%]}\end{array}$ & $\begin{array}{c}\text { Boron } \\
{[\%]}\end{array}$ & $\begin{array}{c}\mathrm{R} \\
{[\%]}\end{array}$ & $\begin{array}{c}\mathrm{E} \\
{[\mathrm{eV}]}\end{array}$ \\
\hline \multirow{2}{*}[\mathrm{S}-\mathrm{BAr}_{4}{}_{4}]{$^{-}$} & LUMO & 5 & 5 & 5 & 5 & 20 & 0 & $80\left(\mathrm{SF}_{5}\right)$ & -1.35 \\
& HOMO & 33 & 33 & 14 & 13 & 93 & 4 & $3\left(\mathrm{SF}_{5}\right)$ & -4.59 \\
& LUMO & 22 & 22 & 23 & 22 & 89 & 1 & $10\left(\mathrm{CF}_{3}\right)$ & 0.25 \\
{$\left[\mathrm{BAr}_{4}\right]^{-}$} & HOMO & 33 & 13 & 33 & 13 & 92 & 5 & $3\left(\mathrm{CF}_{3}\right)$ & -3.62 \\
\hline
\end{tabular}

\subsection{Computational Details}

All DFT geometry optimizations were performed at the GGA BP $86^{3,4}$ level with the Gaussian09 package. ${ }^{5}$ The electronic configuration of the systems was described with the $6-31 \mathrm{G}(\mathrm{d})$ basis set for all atoms. ${ }^{6}$ All geometries were characterized as minimum through frequency calculations. Natural charges were determined by the natural population analysis tool of the Gaussian09 package. Results were analyzed with the Gaussum package ${ }^{7}$ whereas visualization of the results was achieved with avogadro software package. ${ }^{8,9}$ Graphical representations of molecular orbitals were rendered with the help of GNU Paralle ${ }^{10}$ and plotted using the vmd program package ${ }^{11}$ in combination with POV-Ray. ${ }^{12}$

\subsection{Structures}

\subsection{1 $\left[\mathrm{S}-\mathrm{BAr}^{\mathrm{F}}\right]^{-}$}

85

SCF Done: -8125.309715

$\begin{array}{lrrr}\text { S } & 4.427660 & 1.036958 & 3.003516 \\ \text { S } & -4.454570 & -2.875417 & 1.273702 \\ \text { S } & 1.400624 & 3.225893 & -4.233399 \\ \text { S } & 1.213763 & -3.325000 & 4.221654 \\ \text { S } & -1.521504 & -4.171704 & -3.260607 \\ \text { S } & -1.076666 & 4.299111 & 3.265100 \\ \text { S } & 4.254250 & -1.401126 & -3.105178 \\ \text { S } & -4.242506 & 3.214310 & -1.165936 \\ \text { F } & 1.168468 & 4.187082 & -2.914967 \\ \text { F } & 5.745620 & 1.965711 & 3.326183 \\ \text { F } & -5.502157 & -3.029686 & 0.005411 \\ \text { F } & -5.729898 & -3.160909 & 2.271406 \\ \text { F } & 3.567149 & 2.432717 & 2.836119 \\ \text { F } & -4.201662 & -4.508408 & 1.247892 \\ \text { F } & 0.894009 & -4.634068 & 5.164668 \\ \text { F } & 4.117811 & 1.039037 & 4.626740 \\ \text { F } & -1.311635 & 5.697693 & 2.415538\end{array}$

F $\quad 4.849937 \quad 1.109040 \quad 1.412478$

F $\quad-1.839006-5.556299-2.415199$

F $\quad 2.784186-3.837901 \quad 4.167466$

F $\quad 5.397686 \quad-0.285831 \quad 3.202514$

F $\quad 1.176329 \quad 4.563596 \quad-5.163611$

F $\quad-0.740787 \quad 5.236060 \quad 4.574410$

F $\quad-3.514318 \quad-2.749845 \quad 2.621453$

$\begin{array}{llll}\text { F } & 0.529186 & 4.346047 & 2.901891\end{array}$

F $\quad 3.012182 \quad 3.594542 \quad-4.219251$

F $\quad 5.328828 \quad-0.164020 \quad-3.317137$

$\begin{array}{llll}\text { F } & -0.223177 & 2.967635 & -4.329575\end{array}$

$\begin{array}{llll}\text { F } & -0.819876 & 2.984325 & 4.225153\end{array}$

$\begin{array}{llll}\text { F } & 0.860617 & -4.265082 & 2.914682\end{array}$

F $\quad-5.519651 \quad 3.597818 \quad-2.128115$

F $\quad-2.660742 \quad 4.334596 \quad 3.736436$

F $\quad-3.117733 \quad-4.077712 \quad-3.680163$

$\begin{array}{llll}\text { F } & -3.842135 & 4.817554 & -1.179748\end{array}$

$\begin{array}{llll}\text { F } & -4.816962 & -1.271544 & 1.379757\end{array}$

F $\quad 0.086369 \quad-4.349253 \quad-2.951243$ 


\begin{tabular}{|c|c|c|c|}
\hline $\mathrm{F}$ & -5.229291 & 3.484768 & 0.131561 \\
\hline $\mathrm{F}$ & 3.911766 & -1.364735 & -4.721445 \\
\hline $\mathrm{F}$ & 4.702066 & -1.520677 & -1.523961 \\
\hline $\mathrm{F}$ & 5.481427 & -2.436047 & -3.461509 \\
\hline $\mathrm{F}$ & -0.376844 & -2.920514 & 4.359103 \\
\hline$\Gamma$ & 1.546366 & -2.495200 & 5.612346 \\
\hline $\mathrm{F}$ & -3.362773 & 2.981527 & -2.540075 \\
\hline $\mathrm{F}$ & -1.302848 & -5.117920 & -4.587980 \\
\hline F & 1.620719 & 2.376914 & -5.634581 \\
\hline $\mathrm{F}$ & -1.192973 & -2.870743 & -4.217706 \\
\hline $\mathrm{F}$ & -4.751163 & 1.648467 & -1.228479 \\
\hline $\mathrm{F}$ & 3.282099 & -2.720067 & -2.927594 \\
\hline $\mathrm{C}$ & 0.972686 & -0.440424 & 1.268525 \\
\hline $\mathrm{C}$ & 2.880327 & -0.245177 & -2.710107 \\
\hline $\mathrm{C}$ & 2.952668 & -0.000422 & 2.645496 \\
\hline $\mathrm{C}$ & -3.026901 & -2.559257 & 0.159257 \\
\hline $\mathrm{C}$ & -2.813247 & 2.788407 & -0.090821 \\
\hline $\mathrm{C}$ & 1.654959 & 1.729011 & -3.195097 \\
\hline $\mathrm{C}$ & -1.455545 & 3.254030 & 1.799594 \\
\hline $\mathrm{C}$ & 1.574022 & -1.860053 & 3.169158 \\
\hline $\mathrm{C}$ & -0.919625 & 1.313445 & 0.408409 \\
\hline $\mathrm{C}$ & -1.769074 & -3.115695 & -1.775072 \\
\hline $\mathrm{C}$ & 0.976410 & 0.355028 & -1.287179 \\
\hline $\mathrm{C}$ & -1.032740 & -1.241092 & -0.384796 \\
\hline B & -0.001077 & -0.003661 & 0.001057 \\
\hline $\mathrm{C}$ & 2.707666 & -1.101597 & 3.471865 \\
\hline $\mathrm{H}$ & 3.364624 & -1.353695 & 4.302042 \\
\hline $\mathrm{C}$ & 0.718527 & -1.551910 & 2.103882 \\
\hline $\mathrm{H}$ & -0.151769 & -2.184407 & 1.915862 \\
\hline $\mathrm{C}$ & 2.124441 & 0.330068 & 1.567742 \\
\hline $\mathrm{H}$ & 2.373640 & 1.190201 & 0.941153 \\
\hline $\mathrm{C}$ & 2.050758 & -0.509931 & -1.615391 \\
\hline $\mathrm{H}$ & 2.238618 & -1.392128 & -0.997993 \\
\hline $\mathrm{C}$ & 0.799993 & 1.489330 & -2.111873 \\
\hline $\mathrm{H}$ & -0.008065 & 2.193218 & -1.900935 \\
\hline $\mathrm{C}$ & 2.711987 & 0.877692 & -3.526370 \\
\hline $\mathrm{H}$ & 3.369360 & 1.077329 & -4.370283 \\
\hline $\mathrm{C}$ & -0.865700 & -2.078300 & -1.511335 \\
\hline $\mathrm{H}$ & -0.026740 & -1.913609 & -2.190755 \\
\hline $\mathrm{C}$ & -2.148826 & -1.509126 & 0.447114 \\
\hline $\mathrm{H}$ & -2.330453 & -0.878518 & 1.321093 \\
\hline $\mathrm{C}$ & -2.867995 & -3.387516 & -0.956346 \\
\hline $\mathrm{H}$ & -3.562923 & -4.196140 & -1.174319 \\
\hline $\mathrm{C}$ & -0.650275 & 2.142487 & 1.521042 \\
\hline $\mathrm{H}$ & 0.192456 & 1.913784 & 2.176959 \\
\hline $\mathrm{C}$ & -2.034441 & 1.666452 & -0.393207 \\
\hline $\mathrm{H}$ & -2.293770 & 1.047554 & -1.255957 \\
\hline $\mathrm{C}$ & -2.552004 & 3.609924 & 1.010253 \\
\hline $\mathrm{H}$ & -3.169501 & 4.476067 & 1.239925 \\
\hline
\end{tabular}

\subsection{2 $\left[\mathrm{BAr}_{4}{ }_{4}\right]^{-}$}

69

SCF Done -3647.854122

\begin{tabular}{|c|c|c|c|}
\hline & & & \\
\hline & 818 & 57448 & \\
\hline & & & \\
\hline & & & \\
\hline & & & \\
\hline & & & \\
\hline & & & \\
\hline & 2.8 & & \\
\hline & 4.124036 & -3.6 & \\
\hline & & & \\
\hline & & & \\
\hline & 3.6 & & \\
\hline & 0.6 & & \\
\hline & & & -2 \\
\hline & 0.2 & & \\
\hline & -2.6 & & \\
\hline & -2.1 & & \\
\hline & -0.7 & & \\
\hline & -5.3 & & \\
\hline & -6.2 & & \\
\hline & -4.9 & & \\
\hline & -2.3 & & \\
\hline & -2.9 & & \\
\hline & -4.4 & & \\
\hline & -0. & & \\
\hline & & & \\
\hline & -0.2 & & \\
\hline & & & \\
\hline & & & \\
\hline & & & -2 \\
\hline & & & \\
\hline & & & \\
\hline & & & \\
\hline & & & \\
\hline & & & \\
\hline & & & \\
\hline & 3.0 & & \\
\hline & 2.6 & & \\
\hline & 1.6 & & \\
\hline & & & \\
\hline & & & \\
\hline & -1.6 & & \\
\hline & & & \\
\hline & & & \\
\hline & -0.4 & & -0 . \\
\hline & -0.9 & & \\
\hline & -0.8 & 2.4 & 0.78564 \\
\hline
\end{tabular}




$\begin{array}{lrrrrrrr}\mathrm{C} & -0.161472 & 1.640034 & -0.194717 & \mathrm{H} & 2.546528 & 0.027298 & -1.174869 \\ \mathrm{C} & -5.113269 & -0.272986 & -0.858627 & \mathrm{H} & 1.193185 & -2.451329 & 0.673533 \\ \mathrm{C} & -3.188280 & -2.938792 & 2.945381 & \mathrm{H} & 3.754344 & -1.319697 & 3.965336 \\ \mathrm{C} & -2.648557 & -0.194243 & -0.307306 & \mathrm{H} & 1.348796 & 1.665626 & 1.971694 \\ \mathrm{C} & -3.936259 & -0.668488 & -0.003407 & \mathrm{H} & 0.865189 & 1.827438 & -2.098877 \\ \mathrm{C} & -4.132435 & -1.556432 & 1.066474 & \mathrm{H} & -0.584398 & 5.575204 & -0.510598 \\ \mathrm{C} & -3.018253 & -1.952057 & 1.817939 & \mathrm{H} & -1.283548 & 1.897265 & 1.647537 \\ \mathrm{C} & -1.731338 & -1.474436 & 1.499225 & \mathrm{H} & -2.536527 & 0.518184 & -1.132579 \\ \mathrm{C} & -1.499792 & -0.584483 & 0.427437 & \mathrm{H} & -5.134001 & -1.912939 & 1.319218 \\ \mathrm{~B} & -0.011056 & 0.002653 & 0.002238 & \mathrm{H} & -0.890407 & -1.797271 & 2.122507 \\ \mathrm{H} & -1.340729 & -1.676672 & -1.974890 & & & & \\ \mathrm{H} & 1.919934 & -2.350290 & -4.728413 & & & & \end{array}$

\subsection{Graphical Representation of Frontier Orbitals}
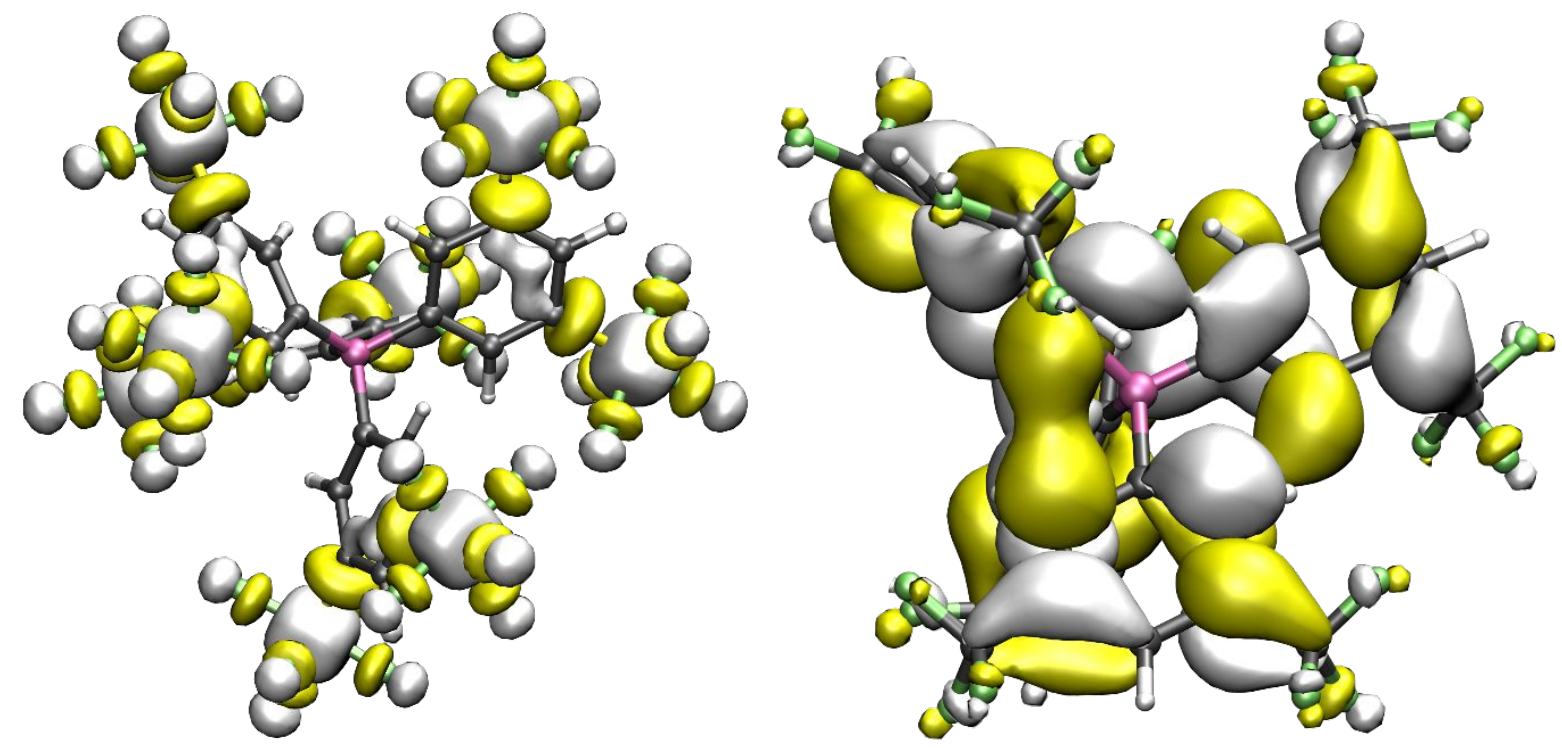

Figure S32: Graphical Representation of the lowest unoccupied MOs of [S-BAr $\left.{ }_{4}\right]^{-}$(left) and $\left[\mathrm{BAr}_{4}\right]^{-}(\operatorname{right})$.
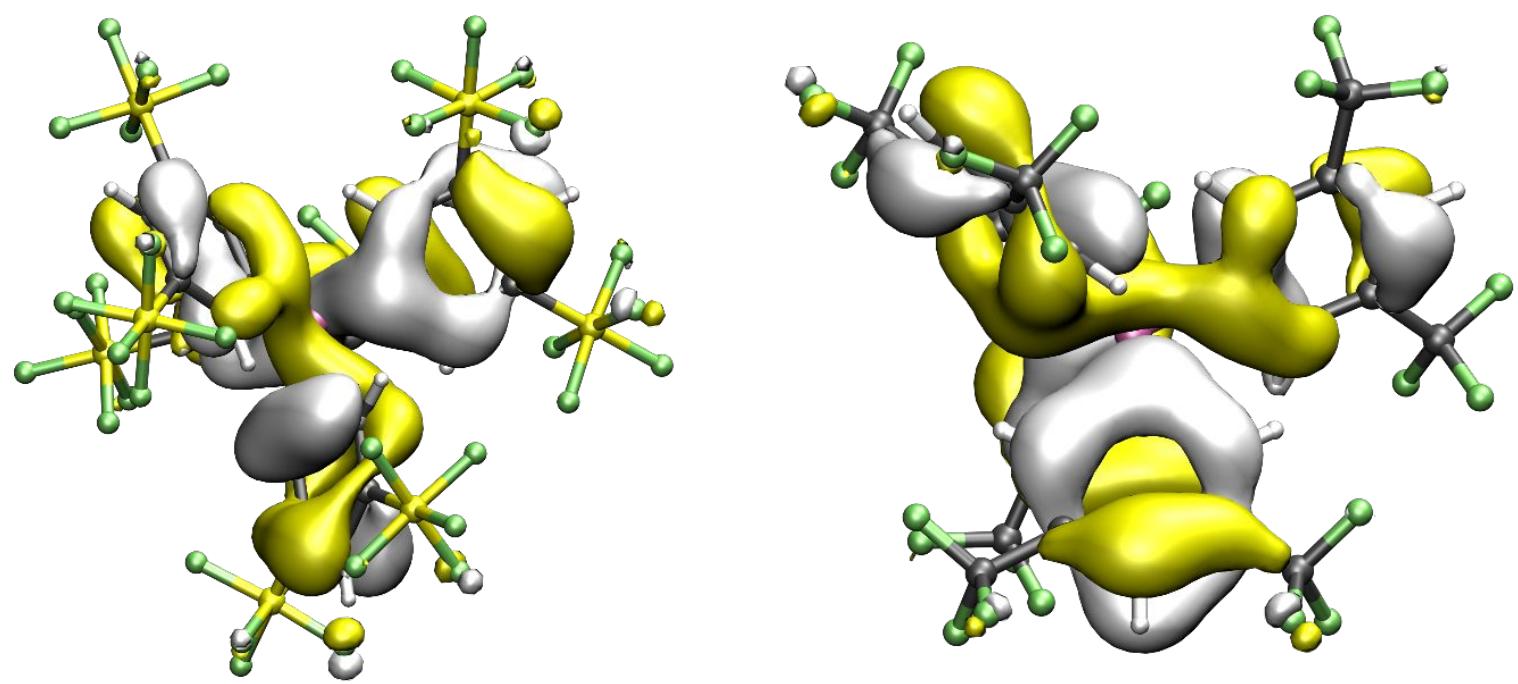

Figure S33: Graphical Representation of the highest occupied MOs of of [S-BAr $\left.{ }_{4}\right]^{-}$(left) and [BAr $\left.{ }_{4}\right]^{-}(\operatorname{right})$. 


\section{Cyclovoltammetric Measurements}

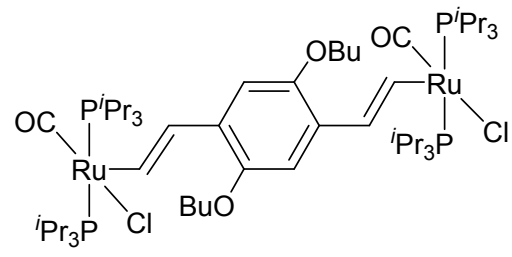

Figure S34: 1,4-divinylphenylene bridged diruthenium complex applied as probe in CV measurements.

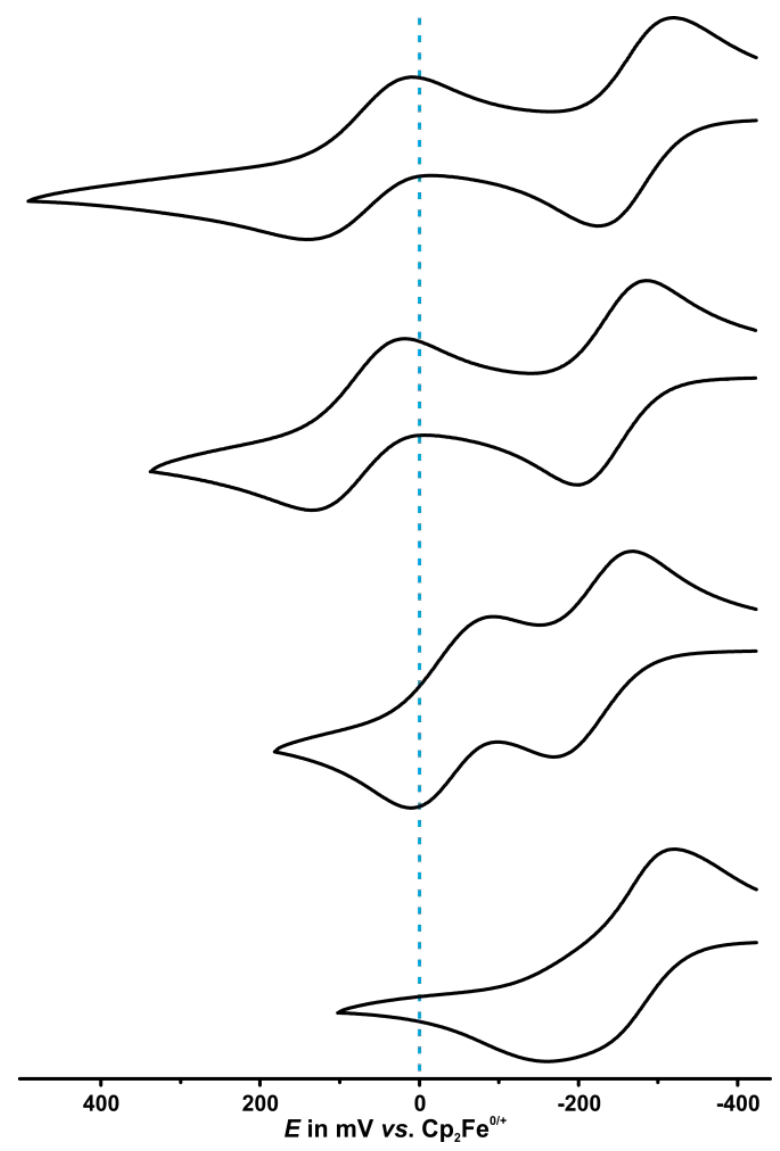

Figure S35: Cyclic voltammograms $\left(v=100 \mathrm{mV} \mathrm{s}^{-1}\right)$ of the probe complex in THF in the presence of $0.1 \mathrm{mM}$ quantities of

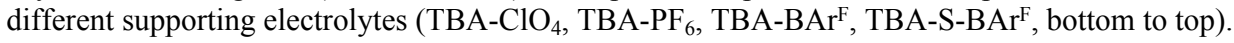

Table S4: Half-wave potential and splitting thereof from cyclovoltammetric measurements.

\begin{tabular}{|c|c|c|c|}
\hline & $\begin{array}{l}\mathrm{E}_{1 / 2}{ }^{0 /+} \\
{[\mathrm{mV}]}\end{array}$ & $\begin{array}{c}\mathrm{E}_{1 / 2}^{+/ 2+} \\
{[\mathrm{mV}]}\end{array}$ & $\begin{array}{l}\Delta \mathrm{E}_{1 / 2} \\
{[\mathrm{mV}]}\end{array}$ \\
\hline TBA-S-BArF & -271 & 74 & 345 \\
\hline TBA-BArF & -262 & 52 & 314 \\
\hline $\mathrm{TBA}^{-\mathrm{PF}_{6}}$ & -228 & -44 & 184 \\
\hline $\mathrm{TBA}_{-} \mathrm{ClO}_{4}$ & \multicolumn{2}{|c|}{-240} & - \\
\hline
\end{tabular}




\section{References}

(1) Yakelis, N. A.; Bergman, R. G.; Organometallics 2005, 24, 3579-3581.

(2) Leicht, H.; Göttker-Schnetmann, I.; Mecking, S.; ACS Macro Lett. 2016, 5, 777-780.

(3) Becke, A. D.; Phys. Rev. A 1988, 38, 3098-3100.

(4) Perdew, J. P.; Phys. Rev. B 1986, Correction. 34, 7406-7406.

(5) Frische, M. J.; Gaussian 09 Revision A. 1, Gaussian Inc., Wallingford, CT, 2009.

(6) Weigend, F.; Ahlrichs, R.; PCCP 2005, 7, 3297-3305.

(7) O'Boyle, N. M.; Tenderholt, A. L.; Langner, K. M.; J. Comput. Chem. 2008, 29, 839-845.

(8) Hanwell, M. D.; Curtis, D. E.; Lonie, D. C.; Vandermeersch, T.; Zurek, E.; Hutchison, G. R.; J. Cheminf. 2012, 4, 17.

(9) Avogadro: an open-source molecular builder and visualization tool. Version 1.2.0. http://avogadro.cc/

(10) Tange, O.; USENIX Mag. 2011, 36, 42-47.

(11) Humphrey, W.; Dalke, A.; Schulten, K.; J. Mol. Graphics 1996, 14, 33-38.

(12) Persistence of Vision Pty. Ltd, Persistence of Vision Raytrace, Version 3.7; Williamstown, Victoria, Australia, 2004. 